\title{
COMPUTING THE CANONICAL HEIGHT ON K3 SURFACES
}

\author{
GREGORY S. CALL AND JOSEPH H. SILVERMAN
}

\begin{abstract}
Let $S$ be a surface in $\mathbb{P}^{2} \times \mathbb{P}^{2}$ given by the intersection of a $(1,1)$ form and a $(2,2)$-form. Then $S$ is a K3 surface with two noncommuting involutions $\sigma^{x}$ and $\sigma^{y}$. In 1991 the second author constructed two height functions $\hat{h}^{+}$and $\hat{h}^{-}$which behave canonically with respect to $\sigma^{x}$ and $\sigma^{y}$, and in 1993 together with the first author showed in general how to decompose such canonical heights into a sum of local heights $\sum_{v} \hat{\lambda}^{ \pm}(\cdot, v)$. We discuss how the geometry of the surface $S$ is related to formulas for the local heights, and we give practical algorithms for computing the involutions $\sigma^{x}, \sigma^{y}$, the local heights $\hat{\lambda}^{+}(\cdot, v), \hat{\lambda}^{-}(\cdot, v)$, and the canonical heights $\hat{h}^{+}, \hat{h}^{-}$.
\end{abstract}

\section{INTRODUCTION}

Let $S \subset \mathbb{P}^{2} \times \mathbb{P}^{2}$ be a K3 surface defined by the vanishing of a $(1,1)$-form $L(\mathbf{x}, \mathbf{y})$ and a $(2,2)$-form $Q(\mathbf{x}, \mathbf{y})$. The two projections $S \rightarrow \mathbb{P}^{2}$ are double covers, so they induce involutions $\sigma^{x}, \sigma^{y}: S \rightarrow S$. The involutions $\sigma^{x}$ and $\sigma^{y}$ are rational maps, and they will be morphisms provided that the projections have no degenerate fibers, that is, no fibers of positive dimension.

Suppose now that $S$ is defined over a number field $K$ and that $\sigma^{x}, \sigma^{y}$ are morphisms. Then Silverman [6] has defined two height functions $\hat{h}^{ \pm}: S(\bar{K}) \rightarrow[0, \infty)$ which behave canonically relative to $\sigma^{x}$ and $\sigma^{y}$. (See Theorem 3.1.) These heights have many interesting arithmetic properties, including the property that

$$
\hat{h}^{+}(P)=0 \Longleftrightarrow \hat{h}^{-}(P)=0 \Longleftrightarrow P \text { has finite orbit under } \sigma^{x} \text { and } \sigma^{y} .
$$

Thus $\hat{h}^{+}$and $\hat{h}^{-}$are analogous to the usual canonical heights on elliptic curves and abelian varieties.

The construction of canonical heights can be extended to even more general settings whenever Tate's telescoping sum construction applies, see [2, Theorem 1.1]. Néron and Tate have shown that the canonical height on an abelian variety can be decomposed into a sum of local height functions, one for each place of $K$, and this construction can also be generalized [2, Theorem 2.1].

The decomposition into local heights offers a more practical method for calculating the canonical height. For non-Archimedean $v$, one can show that if the variety

Received by the editor August 2, 1994.

1991 Mathematics Subject Classification. Primary 11G35, 11Y50, 14G25, 14J20, 14J28.

Key words and phrases. K3 surface, canonical height.

Research of the first author was partially supported by NSF ROA-DMS-8913113, NSA MDA 904-93-H-3022, and an Amherst Trustee Faculty Fellowship.

Research of the second author was partially supported by NSF DMS-9121727. 
and morphism have good reduction modulo $v$, then the canonical local height can be computed as a simple intersection index. It remains to devise a method for computing the canonical local height for non-Archimedean places of bad reduction and for Archimedean places.

Tate [7] described a rapidly convergent series for the canonical local height on the $v$-adic points of an elliptic curve provided that the complete field $K_{v}$ is not algebraically closed, and Silverman [5] gave a modified series which converges with no restriction on $K_{v}$. These constructions were generalized by Call and Silverman $[2, \S 5]$, where they gave a series for the canonical local height on a general variety $V$ with morphism $\phi: V \rightarrow V$. As explained in [2], in order to implement this series in practice, one must explicitly write down certain rational functions whose existence is guaranteed by general principles. Further, one must have an explicit implementation of the morphism $\phi$.

In this paper we will describe how to implement the algorithms in [2] for the K3 surfaces described above. We begin in the first two sections by setting notation and studying the geometry of the surface $S$. In particular, we develop important formulas related to degeneracy of fibers and the involutions $\sigma^{x}$ and $\sigma^{y}$. The third section briefly reviews the theory of canonical heights on $S$ as developed in [2] and [6]. In $\S 4$ we define some error functions and give convergent series for the canonical local heights which are useful theoretically, but not good for practical computations. Next in $\S 5$ we show that if the fibers of $S$ are nondegenerate modulo $v$, then the error functions all vanish, and hence the series from $\S 4$ reduce to a single term. This reduces the computation of $\hat{h}^{+}$and $\hat{h}^{-}$to computing the local height for the places of bad reduction and for the Archimedean places.

The remainder of the paper is concerned with practical computation of these remaining canonical heights. We begin in $\S 6$ by giving an algorithm to compute the involutions $\sigma^{x}$ and $\sigma^{y}$. Then in $\S 7$ we construct the rational functions needed to implement the series [2] for the canonical local height and we describe the resulting algorithm. Finally, in $\S 8$ we consider the particular surface $S$ already studied in $[6$, $\S 5]$. We show how to find the primes of bad reduction, and we implement our algorithms to compute the canonical local and global heights of some of its points. An appendix is included giving the implementation of the algorithms to compute $\sigma^{x}, \sigma^{y}, \hat{\lambda}^{+}(\cdot, v)$ and $\hat{\lambda}^{-}(\cdot, v)$.

\section{Notation AND GeOMETRY}

In this section we will describe the notation which will be used throughout this paper.

$$
\begin{aligned}
K & \text { a field } \\
\mathbf{x}, \mathbf{y} & \text { coordinate functions on } \mathbb{P}^{2} \times \mathbb{P}^{2}, \mathbf{x}=\left[x_{0}, x_{1}, x_{2}\right], \mathbf{y}=\left[y_{0}, y_{1}, y_{2}\right] . \\
L, Q & \text { a }(1,1) \text {-form and a }(2,2) \text {-form defined over } K \text { by } \\
& L(\mathbf{x}, \mathbf{y})=\sum_{i, j=0}^{2} a_{i j} x_{i} y_{j}, \quad Q(\mathbf{x}, \mathbf{y})=\sum_{i, j, k, l=0}^{2} b_{i j k l} x_{i} x_{j} y_{k} y_{l} .
\end{aligned}
$$


$S / K \quad$ the variety $S \subset \mathbb{P}^{2}(K) \times \mathbb{P}^{2}(K)$ defined by $L(\mathbf{x}, \mathbf{y})=Q(\mathbf{x}, \mathbf{y})=$ 0 . We will always assume that $S$ has dimension 2 and that $S$ does not contain a component of the form $\{\mathbf{a}\} \times \mathbb{P}^{2}$ or $\mathbb{P}^{2} \times\{\mathbf{b}\}$. However, unless explicitly stated, we do not assume that $S / K$ is smooth.

$p_{1}, p_{2} \quad$ projections $p_{j}: S \rightarrow \mathbb{P}^{2}$ induced by $p_{j}: \mathbb{P}^{2} \times \mathbb{P}^{2} \rightarrow \mathbb{P}^{2}$.

$\sigma^{x}, \sigma^{y}$ involutions on $S$ induced by the double covers $p_{j}: S \rightarrow \mathbb{P}^{2}$.

$m, n$ indices chosen from $\{0,1,2\}$.

$D_{m}^{x}, D_{n}^{y}$ the divisors $D_{m}^{x}=p_{1}^{*}\left\{x_{m}=0\right\}$ and $D_{n}^{y}=p_{2}^{*}\left\{y_{n}=0\right\}$ in $\operatorname{Div}(S)$.

It is convenient to define linear forms $L_{i}^{*}$ and quadratic forms $Q_{i j}^{*}$ by

$$
\begin{aligned}
L_{j}^{x}(\mathbf{x}) & =\text { the coefficient of } y_{j} \text { in } L(\mathbf{x}, \mathbf{y}), \\
L_{i}^{y}(\mathbf{y}) & =\text { the coefficient of } x_{i} \text { in } L(\mathbf{x}, \mathbf{y}), \\
Q_{k l}^{x}(\mathbf{x}) & =\text { the coefficient of } y_{k} y_{l} \text { in } Q(\mathbf{x}, \mathbf{y}), \\
Q_{i j}^{y}(\mathbf{y}) & =\text { the coefficient of } x_{i} x_{j} \text { in } Q(\mathbf{x}, \mathbf{y}) .
\end{aligned}
$$

This notation allows us to write

$$
\begin{gathered}
L(\mathbf{x}, \mathbf{y})=\sum_{i=0}^{2} L_{i}^{x}(\mathbf{x}) y_{i}=\sum_{i=0}^{2} L_{i}^{y}(\mathbf{y}) x_{i}, \\
Q(\mathbf{x}, \mathbf{y})=\sum_{0 \leq i \leq j \leq 2} Q_{i j}^{x}(\mathbf{x}) y_{i} y_{j}=\sum_{0 \leq i \leq j \leq 2} Q_{i j}^{y}(\mathbf{y}) x_{i} x_{j} .
\end{gathered}
$$

The following quartic forms will appear frequently in our calculations. In these formulas, the indices $(i, j, k)$ are some permutation of $\{0,1,2\}$ and the $*$ may be replaced by either $x$ or $y$.

$$
\begin{aligned}
G_{k}^{*} & =\left(L_{j}^{*}\right)^{2} Q_{i i}^{*}-L_{i}^{*} L_{j}^{*} Q_{i j}^{*}+\left(L_{i}^{*}\right)^{2} Q_{j j}^{*}, \\
H_{i j}^{*} & =2 L_{i}^{*} L_{j}^{*} Q_{k k}^{*}-L_{i}^{*} L_{k}^{*} Q_{j k}^{*}-L_{j}^{*} L_{k}^{*} Q_{i k}^{*}+\left(L_{k}^{*}\right)^{2} Q_{i j}^{*} .
\end{aligned}
$$

Finally we define four sixth-degree forms $R^{x}(\mathbf{X}), R^{y}(\mathbf{Y}), g^{x}(\mathbf{X})$ and $g^{y}(\mathbf{Y})$ by the formulas

$$
\begin{aligned}
R^{*} & =Q_{00}^{*}\left(Q_{12}^{*}\right)^{2}+Q_{11}^{*}\left(Q_{02}^{*}\right)^{2}+Q_{22}^{*}\left(Q_{01}^{*}\right)^{2}-Q_{01}^{*} Q_{02}^{*} Q_{12}^{*}-4 Q_{00}^{*} Q_{11}^{*} Q_{22}^{*}, \\
g^{*} & =\frac{\left(H_{i j}^{*}\right)^{2}-4 G_{i}^{*} G_{j}^{*}}{\left(L_{k}^{*}\right)^{2}}
\end{aligned}
$$

A straightforward calculation shows that $g^{x}(\mathbf{X})$ and $g^{y}(\mathbf{Y})$ are indeed homogeneous polynomials and that their definition is independent of the ordering of $(i, j, k)$. More precisely, one can verify that

$$
\begin{aligned}
g^{*}= & L_{0}^{* 2} Q_{12}^{*}+L_{1}^{* 2} Q_{02}^{*}+L_{2}^{* 2} Q_{01}^{*}{ }^{2} \\
& -2 L_{0}^{*} L_{1}^{*} Q_{02}^{*} Q_{12}^{*}-2 L_{0}^{*} L_{2}^{*} Q_{01}^{*} Q_{12}^{*}-2 L_{1}^{*} L_{2}^{*} Q_{01}^{*} Q_{02}^{*} \\
& +4 L_{0}^{*} L_{1}^{*} Q_{01}^{*} Q_{22}^{*}+4 L_{0}^{*} L_{2}^{*} Q_{02}^{*} Q_{11}^{*}+4 L_{1}^{*} L_{2}^{*} Q_{12}^{*} Q_{00}^{*} \\
& -4 L_{0}^{* 2} Q_{11}^{*} Q_{22}^{*}-4 L_{1}^{* 2} Q_{00}^{*} Q_{22}^{*}-4 L_{2}^{* 2} Q_{11}^{*} Q_{00}^{*}
\end{aligned}
$$


We call $g^{x}$ and $g^{y}$ the ramification polynomials of $\sigma^{x}$ and $\sigma^{y}$ respectively. See Proposition 2.1 below for the appropriateness of this name.

For any given points $\mathbf{a}, \mathbf{b} \in \mathbb{P}^{2}$ we denote various fibers as follows:

$$
\begin{aligned}
L_{\mathbf{a}}^{x} & =\left\{(\mathbf{a}, \mathbf{y}) \in \mathbb{P}^{2} \times \mathbb{P}^{2}: L(\mathbf{a}, \mathbf{y})=0\right\}, \\
Q_{\mathbf{a}}^{x} & =\left\{(\mathbf{a}, \mathbf{y}) \in \mathbb{P}^{2} \times \mathbb{P}^{2}: Q(\mathbf{a}, \mathbf{y})=0\right\}, \\
S_{\mathbf{a}}^{x} & =p_{1}^{-1}(\mathbf{a})=L_{\mathbf{a}}^{x} \cap Q_{\mathbf{a}}^{x}, \\
L_{\mathbf{b}}^{y} & =\left\{(\mathbf{x}, \mathbf{b}) \in \mathbb{P}^{2} \times \mathbb{P}^{2}: L(\mathbf{x}, \mathbf{b})=0\right\}, \\
Q_{\mathbf{b}}^{y} & =\left\{(\mathbf{x}, \mathbf{b}) \in \mathbb{P}^{2} \times \mathbb{P}^{2}: Q(\mathbf{x}, \mathbf{b})=0\right\}, \\
S_{\mathbf{b}}^{y} & =p_{2}^{-1}(\mathbf{b})=L_{\mathbf{b}}^{y} \cap Q_{\mathbf{b}}^{y} .
\end{aligned}
$$

To ease notation, we will often write $\mathbf{y} \in L_{\mathbf{a}}^{x}$ rather than $(\mathbf{a}, \mathbf{y}) \in L_{\mathbf{a}}^{x}$.

We begin with the following elementary result, where we recall that the rank of a bilinear form such as $L$ is defined to be the rank of the associated $3 \times 3$ matrix $\left(a_{i j}\right)$.

Lemma 1.1. (a) The following four conditions are equivalent:

(i) There exists an $\mathbf{a} \in \mathbb{P}^{2}(K)$ such that $L_{\mathbf{a}}^{x}(Y) \equiv 0$.

(ii) There exists $a \mathbf{b} \in \mathbb{P}^{2}(K)$ such that $L_{\mathbf{b}}^{y}(X) \equiv 0$.

(iii) $\operatorname{rank} L \leq 2$.

(iv) The locus of $L(\mathbf{x}, \mathbf{y})=0$ is singular in $\mathbb{P}^{2} \times \mathbb{P}^{2}$.

(b) If $S$ is smooth, then $\operatorname{rank} L \geq 2$.

Proof. (a) Let $A=\left(a_{i j}\right)$ be the matrix associated with $L$. Condition (i) says that the columns of $A$ are linearly dependent, and (ii) says that the rows are dependent, so (i), (ii) and (iii) are equivalent. Further, since $L_{j}^{x}=\partial L / \partial y_{j}$ and $L_{i}^{y}=\partial L / \partial x_{i}$, we see that the locus $L=0$ has a singular point if and only if both (i) and (ii) are true.

(b) If $L$ has rank less than 2 , then there are lines $l_{1}$ and $l_{2}$ in $\mathbb{P}^{2}$ such that $L(\mathbf{a}, \mathbf{Y}) \equiv 0$ for all $\mathbf{a} \in l_{1}$ and $L(\mathbf{X}, \mathbf{b}) \equiv 0$ for all $\mathbf{b} \in l_{2}$. Fix some $\mathbf{a} \in l_{1}$. Let $\mathbf{b} \in \mathbb{P}^{2}$ be an intersection point of the line $l_{2}$ and the curve $Q(\mathbf{a}, \mathbf{Y})=0$. Then $(\mathbf{a}, \mathbf{b}) \in S$ and

$$
\frac{\partial L}{\partial x_{i}}(\mathbf{a}, \mathbf{b})=L_{i}^{y}(\mathbf{b})=0 \quad \text { and } \quad \frac{\partial L}{\partial y_{j}}(\mathbf{a}, \mathbf{b})=L_{j}^{x}(\mathbf{a})=0 .
$$

Hence $(\mathbf{a}, \mathbf{b})$ is a singular point of $S$.

For most points $\mathbf{a}$ and $\mathbf{b}$, the fibers $S_{\mathbf{a}}^{x}=p_{1}^{-1}(\mathbf{a})$ and $S_{\mathbf{b}}^{y}=p_{2}^{-1}(\mathbf{b})$ each consists of two points. We will say that a fiber is degenerate if it has positive dimension, and that it is nondegenerate if it consists of a finite set of points. Notice that the projections $p_{1}$ and $p_{2}$ are flat if and only if they have no degenerate fibers. The next proposition tells us that if $S$ is smooth, then the flatness of $p_{1}$ and $p_{2}$ is equivalent to the condition that the rational maps $\sigma^{x}$ and $\sigma^{y}$ are morphisms.

Proposition 1.2. Suppose that $S$ is smooth. Then the projections $p_{1}$ and $p_{2}$ are flat if and only if the maps $\sigma^{x}$ and $\sigma^{y}$ are morphisms of $S$ to itself. 
Proof. Suppose first that $p_{1}$ is flat. Then for each $\mathbf{a} \in \mathbb{P}^{2}$, the fiber $S_{\mathbf{a}}^{x}$ has dimension 0 , so

$$
\operatorname{dim} L_{\mathbf{a}}^{x}=\operatorname{dim} Q_{\mathbf{a}}^{x}=1 \quad \text { and } \quad L_{\mathbf{a}}^{x} \nsubseteq Q_{\mathbf{a}}^{x} .
$$

It follows that $S_{\mathbf{a}}^{x}=L_{\mathbf{a}}^{x} \cap Q_{\mathbf{a}}^{x}$ consists of exactly two points (counted with multiplicity), and hence $\sigma^{x}$ is a morphism. Similarly, if $p_{2}$ is flat, then $\sigma^{y}$ is a morphism.

To prove the converse, we assume that $\sigma^{x}$ and $\sigma^{y}$ are both morphisms. By symmetry, it suffices to prove that $p_{1}$ is flat. Suppose first that some fiber $S_{\mathrm{a}}^{x}$ has dimension 2. This means that $S_{\mathbf{a}}^{x}=\{a\} \times \mathbb{P}^{2}$, contradicting the assumption that $S$ has no components of this form. Hence the fibers have dimension at most 1.

Next suppose that $S_{\mathbf{a}}^{x}$ has dimension 1. Under our assumption that $\sigma^{x}$ and $\sigma^{y}$ are morphisms, it follows from [6, Proposition 2.5] that the divisor $4 D_{m}^{x}-D_{n}^{y}$ is ample. On the other hand, we have

$$
\left(D_{m}^{x}\right) \cdot\left(S_{\mathbf{a}}^{x}\right)=0 \quad \text { and } \quad\left(D_{n}^{y}\right) \cdot\left(S_{\mathbf{a}}^{x}\right)>0, \quad \text { and hence } \quad\left(4 D_{m}^{x}-D_{n}^{y}\right) \cdot\left(S_{\mathbf{a}}^{x}\right)<0 .
$$

But an ample divisor and a positive divisor always intersect positively. This contradiction shows that $S_{\mathbf{a}}^{x}$ has dimension 0 , which concludes the proof that $p_{1}$ is flat.

Corollary 1.3. If $\sigma^{x}$ and $\sigma^{y}$ are automorphisms of $S$ and if $S$ has at least one degenerate fiber, then $S$ is singular.

In view of Proposition 1.2 and Corollary 1.3, it becomes important to determine which fibers are degenerate. The next proposition gives a criterion in terms of the $G_{i}^{*}$ and $H_{i j}^{*}$ forms defined earlier.

Proposition 1.4. Let $(\mathbf{a}, \mathbf{b}) \in S$.

(a) $S_{\mathbf{a}}^{x}$ is a degenerate fiber if and only if

$$
G_{0}^{x}(\mathbf{a})=G_{1}^{x}(\mathbf{a})=G_{2}^{x}(\mathbf{a})=H_{01}^{x}(\mathbf{a})=H_{02}^{x}(\mathbf{a})=H_{12}^{x}(\mathbf{a})=0 .
$$

(b) $S_{\mathbf{b}}^{y}$ is a degenerate fiber if and only if

$$
G_{0}^{y}(\mathbf{b})=G_{1}^{y}(\mathbf{b})=G_{2}^{y}(\mathbf{b})=H_{01}^{y}(\mathbf{b})=H_{02}^{y}(\mathbf{b})=H_{12}^{y}(\mathbf{b})=0 .
$$

Proof. By symmetry, it suffices to prove (a). The surface $S$ is defined by the two equations $L(\mathbf{x}, \mathbf{y})=Q(\mathbf{x}, \mathbf{y})=0$. If we write $y_{0}=\left(L-L_{1}^{x} y_{1}-L_{2}^{x} y_{2}\right) / L_{0}^{x}$, substitute into $Q$, and do a little algebra, we obtain an identity of the form

$$
\begin{aligned}
\left(L_{0}^{x}\right)^{2} Q= & G_{2}^{x} y_{1}^{2}+H_{12}^{x} y_{1} y_{2}+G_{1}^{x} y_{2}^{2} \\
& +L\left\{Q_{00}^{x} L+\left(\left(L_{0}^{x} Q_{01}^{x}-2 L_{1}^{x} Q_{00}^{x}\right) y_{1}+\left(L_{0}^{x} Q_{02}^{x}-2 L_{2}^{x} Q_{00}^{x}\right) y_{2}\right)\right\} .
\end{aligned}
$$

There are analogous formulas obtained by eliminating $y_{1}$ and $y_{2}$. Since we will generally be interested in studying points satisfying at least $L(\mathbf{x}, \mathbf{y})=0$, we will write these three identities as congruences in the polynomial ring $\mathbb{Z}\left[a_{i j}, b_{i j k l}, x_{i}, y_{i}\right]$ as follows:

$$
\begin{aligned}
& L_{0}^{x}(\mathbf{x})^{2} Q(\mathbf{x}, \mathbf{y}) \equiv G_{2}^{x}(\mathbf{x}) y_{1}^{2}+H_{12}^{x}(\mathbf{x}) y_{1} y_{2}+G_{1}^{x}(\mathbf{x}) y_{2}^{2}(\bmod L(\mathbf{x}, \mathbf{y})), \\
& L_{1}^{x}(\mathbf{x})^{2} Q(\mathbf{x}, \mathbf{y}) \equiv G_{2}^{x}(\mathbf{x}) y_{0}^{2}+H_{02}^{x}(\mathbf{x}) y_{0} y_{2}+G_{0}^{x}(\mathbf{x}) y_{2}^{2}(\bmod L(\mathbf{x}, \mathbf{y})), \\
& L_{2}^{x}(\mathbf{x})^{2} Q(\mathbf{x}, \mathbf{y}) \equiv G_{1}^{x}(\mathbf{x}) y_{0}^{2}+H_{01}^{x}(\mathbf{x}) y_{0} y_{1}+G_{0}^{x}(\mathbf{x}) y_{1}^{2}(\bmod L(\mathbf{x}, \mathbf{y})) .
\end{aligned}
$$


Now let $(\mathbf{a}, \mathbf{b}) \in S$. If $L(\mathbf{a}, \mathbf{y})=0$, then $S_{\mathbf{a}}^{x}=Q_{\mathbf{a}}^{x}$, so $S_{\mathbf{a}}^{x}$ is degenerate. Further, (1) and (2) imply that $G_{k}^{x}(\mathbf{a})=H_{i j}^{x}(\mathbf{a})=0$ for all $i, j, k$, so we are done in this case. We now assume that $L(\mathbf{a}, \mathbf{y}) \neq 0$.

Suppose first that $G_{i}^{x}(\mathbf{a})=H_{i j}^{x}(\mathbf{a})=0$ for all $i, j$. We evaluate $(6),(7)$, and (8) at $\mathbf{x}=\mathbf{a}$ and note that one of the $L_{i}^{x}(\mathbf{a})^{\prime}$ 's is nonzero. Hence we must have

$$
Q(\mathbf{a}, \mathbf{y}) \equiv 0 \quad(\bmod L(\mathbf{a}, \mathbf{y})), \quad \text { and so } \quad L_{\mathbf{a}}^{x} \subset Q_{\mathbf{a}}^{x}
$$

In other words, the fiber $S_{\mathbf{a}}^{x}$ contains the entire line $L_{\mathbf{a}}^{x}$, so it is degenerate.

Conversely, suppose that $S_{\mathbf{a}}^{x}$ is degenerate. Under our assumption that $L(\mathbf{a}, \mathbf{Y}) \not \equiv$ 0 , this implies that $S_{\mathbf{a}}^{x}=L_{\mathbf{a}}^{x} \subset Q_{\mathbf{a}}^{x}$. In other words, $Q(\mathbf{a}, \mathbf{y})=0$ for all $\mathbf{y} \in L_{\mathbf{a}}^{x}$. We start by showing that $G_{0}^{x}(\mathbf{a})=G_{1}^{x}(\mathbf{a})=G_{2}^{x}(\mathbf{a})=0$. If $L_{1}^{x}(\mathbf{a})=L_{2}^{x}(\mathbf{a})=0$, then $G_{0}^{x}(\mathbf{a})=0$ directly from the definition $(1)$. If $L_{1}^{x}(\mathbf{a}) \neq 0$ or $L_{2}^{x}(\mathbf{a}) \neq 0$, then $\mathbf{y}_{0}=\left[0, L_{2}^{x}(\mathbf{a}),-L_{1}^{x}(\mathbf{a})\right] \in L_{\mathbf{a}}^{x}$, and we have

(9) $0=Q\left(\mathbf{a}, \mathbf{y}_{0}\right)=Q_{11}^{x}(\mathbf{a}) L_{2}^{x}(\mathbf{a})^{2}-Q_{12}^{x}(\mathbf{a}) L_{1}^{x}(\mathbf{a}) L_{2}^{x}(\mathbf{a})+Q_{22}^{x}(\mathbf{a}) L_{1}^{x}(\mathbf{a})^{2}=G_{0}^{x}(\mathbf{a})$.

A similar argument shows that $G_{1}^{x}(\mathbf{a})=G_{2}^{x}(\mathbf{a})=0$.

Evaluating (6), (7), and (8) at $\mathbf{x}=\mathbf{a}$ and substituting $G_{0}^{x}(\mathbf{a})=G_{1}^{x}(\mathbf{a})=G_{2}^{x}(\mathbf{a})=$ 0 gives

$$
H_{12}^{x}(\mathbf{a}) y_{1} y_{2}=H_{02}^{x}(\mathbf{a}) y_{0} y_{2}=H_{01}^{x}(\mathbf{a}) y_{0} y_{1}=0
$$

for all points $\mathbf{y}=\left[y_{0}, y_{1}, y_{2}\right] \in L_{\mathbf{a}}^{x}$. By symmetry, it suffices to check that $H_{12}^{x}(\mathbf{a})=$ 0 . If $L_{\mathbf{a}}^{x}$ contains a point with $y_{1} y_{2} \neq 0$, then (10) gives the desired result. Otherwise, $L_{\mathbf{a}}^{x}$ must be one of the two lines $y_{1}=0$ or $y_{2}=0$. If $L_{\mathbf{a}}^{x}$ is the line $y_{1}=0$, then $L_{0}^{x}(\mathbf{a})=L_{2}^{x}(\mathbf{a})=0$, so $H_{12}^{x}(\mathbf{a})=0$ from the definition $(2)$; and similarly if $L_{\mathbf{a}}^{x}$ is the line $y_{2}=0$.

We are now ready to give formulas for computing the automorphisms $\sigma^{x}$ and $\sigma^{y}$ on degenerate fibers. These formulas will be useful for theoretical work. We will describe a somewhat more practical algorithm for computing $\sigma^{x}$ and $\sigma^{y}$ automorphisms in $\S 6$.

Corollary 1.5. Fix a point $P=(\mathbf{a}, \mathbf{b}) \in S$.

(a) Suppose that $S_{\mathbf{a}}^{x}$ is a nondegenerate fiber, and write $\sigma^{x} P=\left(\mathbf{a}, \mathbf{b}^{\prime}\right)$. Then $\mathbf{b}, \mathbf{b}^{\prime}$ are the unique points on $L_{\mathbf{a}}^{x}$ defined by the three simultaneous equations

$$
G_{k}^{x}(\mathbf{a}) Y_{l}^{2}+H_{k l}^{x}(\mathbf{a}) Y_{k} Y_{l}+G_{l}^{x}(\mathbf{a}) Y_{k}^{2}=0, \quad(k, l) \in\{(0,1),(0,2),(1,2)\} .
$$

For each such pair $(k, l)$, the coordinates of $P$ and $\sigma^{x} P$ satisfy the relation $\left(\right.$ in $\left.\mathbb{P}^{2}\right)$

$$
\left[b_{k} b_{k}^{\prime}, b_{k} b_{l}^{\prime}+b_{k}^{\prime} b_{l}, b_{l} b_{l}^{\prime}\right]=\left[G_{k}^{x}(\mathbf{a}),-H_{k l}^{x}(\mathbf{a}), G_{l}^{x}(\mathbf{a})\right]
$$

(b) Suppose that $S_{\mathbf{b}}^{y}$ is a nondegenerate fiber, and write $\sigma^{y} P=\left(\mathbf{a}^{\prime}, \mathbf{b}\right)$. Then $\mathbf{a}, \mathbf{a}^{\prime}$ are the unique points on $L_{\mathbf{b}}^{y}$ defined by the three simultaneous equations

$$
G_{i}^{y}(\mathbf{b}) X_{j}^{2}+H_{i j}^{y}(\mathbf{b}) X_{i} X_{j}+G_{j}^{y}(\mathbf{b}) X_{i}^{2}=0, \quad(i, j) \in\{(0,1),(0,2),(1,2)\} .
$$



$\left.\mathbb{P}^{2}\right)$

For each such pair $(i, j)$, the coordinates of $P$ and $\sigma^{y} P$ satisfy the relation (in

$$
\left[a_{i} a_{i}^{\prime}, a_{i} a_{j}^{\prime}+a_{i}^{\prime} a_{j}, a_{j} a_{j}^{\prime}\right]=\left[G_{i}^{y}(\mathbf{b}),-H_{i j}^{y}(\mathbf{b}), G_{j}^{y}(\mathbf{b})\right] .
$$

Proof. By symmetry, it suffices to prove (a). Since $S_{\mathbf{a}}^{x}$ is nondegenerate, $\sigma^{x}$ is well defined. More precisely, $\mathbf{b}$ and $\mathbf{b}^{\prime}$ are defined to be the unique points in $S_{\mathbf{a}}^{x}=L_{\mathbf{a}}^{x} \cap Q_{\mathbf{a}}^{x}$. Hence, our assertion is an immediate consequence of the identities (6), (7), and (8).

\section{Singular POINTS, DEgENERATE FIBERS AND RAMifiCATION POINTS}

In this section we will study the relationship between singular points on the surface $S$, degenerate fibers of the projections $p_{i}: S \rightarrow \mathbb{P}^{2}$, and the ramification loci of these projections. We begin by showing that the ramification polynomials $g^{x}$ and $g^{y}$ defined earlier actually capture the ramification locus of the projections.

Proposition 2.1. Let $P=(\mathbf{a}, \mathbf{b}) \in S$.

(a) $g^{x}(\mathbf{a})=0$ if and only if either $S_{\mathbf{a}}^{x}$ is degenerate or $\sigma^{x}(P)=P$.

(b) $g^{y}(\mathbf{b})=0$ if and only if either $S_{\mathbf{b}}^{y}$ is degenerate or $\sigma^{y}(P)=P$.

Proof. This follows directly from Proposition 1.4, Corollary 1.5, and the definition of $g^{x}$ and $g^{y}$.

Notice that the condition $\sigma^{x}(P)=P$ says precisely that the projection $p_{1}$ : $S \rightarrow \mathbb{P}^{2}$ is ramified over a. Thus, Proposition 2.1 implies that $g^{x}(\mathbf{x})=0$ is the ramification locus of $p_{1}$, and similarly $g^{y}(\mathbf{y})=0$ is the ramification locus of $p_{2}$. We next verify that the degenerate fibers lie above singular points of the ramification locus.

Proposition 2.2. Let $P=(\mathbf{a}, \mathbf{b}) \in S$.

(a) If $S_{\mathbf{a}}^{x}$ is degenerate, then the ramification curve $g^{x}(\mathbf{x})=0$ is singular at $\mathbf{x}=\mathbf{a}$.

(b) If $S_{\mathbf{b}}^{y}$ is degenerate, then the ramification curve $g^{y}(\mathbf{y})=0$ is singular at $\mathbf{y}=\mathbf{b}$.

Proof. Suppose that $S_{\mathbf{a}}^{x}$ is degenerate. Then Proposition 1.4 tells us that $G_{i}^{x}(\mathbf{a})=$ $H_{i j}^{x}(\mathbf{a})=0$ for all $i, j$. If at least one of $L_{0}^{x}(\mathbf{a}), L_{1}^{x}(\mathbf{a})$ and $L_{2}^{x}(\mathbf{a})$ is nonzero, then the defining equation (4) for $g^{x}$ tells us that

$$
\frac{\partial g^{x}}{\partial x_{0}}(\mathbf{a})=\frac{\partial g^{x}}{\partial x_{1}}(\mathbf{a})=\frac{\partial g^{x}}{\partial x_{2}}(\mathbf{a})=0
$$

Otherwise the alternative formula (5) for $g^{x}$ gives us the same result. Hence $\mathbf{a}$ is a singular point of the ramification curve. This proves (a), and (b) is proven similarly.

Next we describe those a's and b's for which the curves $Q_{\mathbf{a}}^{x}$ and $Q_{\mathbf{b}}^{y}$ are smooth.

Lemma 2.3. Let $P=(\mathbf{a}, \mathbf{b}) \in S$.

(a) $Q_{\mathbf{a}}^{x}$ is a smooth curve if and only if $R^{x}(\mathbf{a}) \neq 0$.

(b) $Q_{\mathbf{b}}^{y}$ is a smooth curve if and only if $R^{y}(\mathbf{b}) \neq 0$. 
Proof. By symmetry, it suffices to prove (a). Recall that the curve $Q_{\mathbf{a}}^{x}$ is given by the equation $Q(\mathbf{a}, \mathbf{y})=0$. Suppose first that $\operatorname{char}(K) \neq 2$. Then $Q_{\mathbf{a}}^{x}$ is singular if and only if there is a point $\mathbf{b}=\left[b_{0}, b_{1}, b_{2}\right] \in \mathbb{P}^{2}$ such that

$$
\begin{aligned}
& \frac{\partial Q(\mathbf{a}, \mathbf{y})}{\partial y_{0}}(\mathbf{b})=2 Q_{00}^{x}(\mathbf{a}) b_{0}+Q_{01}^{x}(\mathbf{a}) b_{1}+Q_{02}^{x}(\mathbf{a}) b_{2}=0, \\
& \frac{\partial Q(\mathbf{a}, \mathbf{y})}{\partial y_{1}}(\mathbf{b})=Q_{01}^{x}(\mathbf{a}) b_{0}+2 Q_{11}^{x}(\mathbf{a}) b_{1}+Q_{12}^{x}(\mathbf{a}) b_{2}=0, \\
& \frac{\partial Q(\mathbf{a}, \mathbf{y})}{\partial y_{2}}(\mathbf{b})=Q_{02}^{x}(\mathbf{a}) b_{0}+Q_{12}^{x}(\mathbf{a}) b_{1}+2 Q_{22}^{x}(\mathbf{a}) b_{2}=0 .
\end{aligned}
$$

These linear equations have a solution in $\mathbb{P}^{2}$ if and only if

$$
\operatorname{det}\left(\begin{array}{rrr}
2 Q_{00}^{x}(\mathbf{a}) & Q_{01}^{x}(\mathbf{a}) & Q_{02}^{x}(\mathbf{a}) \\
Q_{01}^{x}(\mathbf{a}) & 2 Q_{11}^{x}(\mathbf{a}) & Q_{12}^{x}(\mathbf{a}) \\
Q_{02}^{x}(\mathbf{a}) & Q_{12}^{x}(\mathbf{a}) & 2 Q_{22}^{x}(\mathbf{a})
\end{array}\right)=-2 R^{x}(\mathbf{a}) \neq 0 .
$$

Now suppose that $\operatorname{char}(K)=2$. If $Q_{01}^{x}(\mathbf{a})=Q_{02}^{x}(\mathbf{a})=Q_{12}^{x}(\mathbf{a})=0$, then $R^{x}(\mathbf{a})=$ 0 directly from the definition (3), and all three partial derivatives $\partial Q(\mathbf{a}, \mathbf{y}) / \partial y_{i}$ are identically zero from (11). Otherwise, the point $\mathbf{b}_{0}=\left[Q_{12}^{x}(\mathbf{a}), Q_{02}^{x}(\mathbf{a}), Q_{01}^{x}(\mathbf{a})\right]$ is the unique point in $\mathbb{P}^{2}$ such that all three partial derivatives (11) vanish. Since $Q\left(\mathbf{a}, \mathbf{b}_{0}\right)=R^{x}(\mathbf{a})$, we conclude that $Q_{\mathbf{a}}^{x}$ is singular if and only if $R^{x}(\mathbf{a})=0$.

Lemma 2.4. Let $P=(\mathbf{a}, \mathbf{b}) \in S$.

(a) If $S_{\mathbf{a}}^{x}$ is degenerate, then either $L(\mathbf{a}, \mathbf{Y}) \equiv 0$ or $R^{x}(\mathbf{a})=0$.

(b) If $S_{\mathbf{b}}^{y}$ is degenerate, then either $L(\mathbf{X}, \mathbf{b}) \equiv 0$ or $R^{y}(\mathbf{b})=0$.

Proof. By symmetry, it suffices to prove (a). Suppose that $S_{\mathbf{a}}^{x}$ is degenerate and that $L(\mathbf{a}, \mathbf{Y}) \not \equiv 0$. Then $L(\mathbf{a}, \mathbf{Y})$ must divide $Q(\mathbf{a}, \mathbf{Y})$, so the curve $Q_{\mathbf{a}}^{x}$ is reducible, hence singular. It follows from Lemma 2.3 that $R^{x}(\mathbf{a})=0$.

Proposition 2.5. Suppose that $P=(\mathbf{a}, \mathbf{b}) \in S$ is a singular point of $S$. Then $g^{x}(\mathbf{a})=g^{y}(\mathbf{b})=0$. In other words, a singular point lies above both of the ramification loci.

Proof. By symmetry, it suffices to prove that $g^{y}(\mathbf{b})=0$. We begin by deriving some new identities. For each pair $(i, j)$ with $0 \leq i, j \leq 2$, let $M_{i j}$ denote the matrix of partial derivatives

$$
M_{i j}=\left(\begin{array}{ll}
\left(\partial L / \partial x_{i}\right)(\mathbf{a}, \mathbf{b}) & \left(\partial L / \partial x_{j}\right)(\mathbf{a}, \mathbf{b}) \\
\left(\partial Q / \partial x_{i}\right)(\mathbf{a}, \mathbf{b}) & \left(\partial Q / \partial x_{j}\right)(\mathbf{a}, \mathbf{b})
\end{array}\right) .
$$

A little algebra gives the identity

$$
L_{i}^{y}(\mathbf{b}) \operatorname{det}\left(M_{i j}\right)=2 a_{j} G_{k}^{y}(\mathbf{b})+a_{k} H_{j k}^{y}(\mathbf{b}),
$$

valid for all $i, j, k$ such that $\{i, j, k\}=\{0,1,2\}$.

If $L_{0}^{y}(\mathbf{b})=L_{1}^{y}(\mathbf{b})=L_{2}^{y}(\mathbf{b})=0$, then $g^{y}(\mathbf{b})=0$ from (5). So we may assume (by symmetry) that $L_{2}^{y}(\mathbf{b}) \neq 0$, and now (4) says that we must verify that $H_{01}^{y}(\mathbf{b})^{2}=$ $4 G_{0}^{y}(\mathbf{b}) G_{1}^{y}(\mathbf{b})$. 
By assumption, the point $P=(\mathbf{a}, \mathbf{b})$ is a singular point of $S$, so $\operatorname{det}\left(M_{i j}\right)=0$ for all $i, j$. Evaluating (12) for various $i, j, k$ 's gives

$$
\begin{aligned}
& 2 a_{0} G_{1}^{y}(\mathbf{b})+a_{1} H_{01}^{y}(\mathbf{b})=0, \\
& 2 a_{1} G_{0}^{y}(\mathbf{b})+a_{0} H_{01}^{y}(\mathbf{b})=0 .
\end{aligned}
$$

It follows either that $H_{01}^{y}(\mathbf{b})^{2}-4 G_{0}^{y}(\mathbf{b}) G_{1}^{y}(\mathbf{b})=0$, in which case we are done, or else that $a_{0}=a_{1}=0$. But if $a_{0}=a_{1}=0$, then $a_{2} \neq 0$, and the equation

$$
0=L(\mathbf{a}, \mathbf{b})=L_{0}^{y}(\mathbf{b}) a_{0}+L_{1}^{y}(\mathbf{b}) a_{1}+L_{2}^{y}(\mathbf{b}) a_{2}=L_{2}^{y}(\mathbf{b}) a_{2}
$$

contradicts our assumption that $L_{2}^{y}(\mathbf{b}) \neq 0$.

Corollary 2.6. Suppose that $P=(\mathbf{a}, \mathbf{b}) \in S$ is a singular point of $S$.

(a) If $L(\mathbf{a}, \mathbf{Y}) \neq \equiv$ and $R^{x}(\mathbf{a}) \neq 0$, then $\sigma^{x}(P)=P$.

(b) If $L(\mathbf{X}, \mathbf{b}) \not \equiv 0$ and $R^{y}(\mathbf{b}) \neq 0$, then $\sigma^{y}(P)=P$.

Proof. This follows immediately from Proposition 2.1, Lemma 2.4 and Proposition 2.5 .

\section{Canonical global and local heights on $S$}

To the notation and hypotheses of the first two sections we make the following additions:

$K \quad$ a field with a complete set of proper absolute values $M_{K}$ satisfying the product formula, see [3]. We will call such a field a global height field, since it is for such fields that one can define a height function on $\mathbb{P}^{n}(\bar{K})$.

$S / K \quad$ we will henceforth assume that $S / K$ is smooth and irreducible and has no degenerate fibers, so $\sigma^{x}$ and $\sigma^{y}$ are automorphisms of $S$ from Proposition 1.2.

$\phi, \psi \quad$ automorphisms of $S$ given by $\phi=\sigma^{y} \circ \sigma^{x}$ and $\psi=\sigma^{x} \circ \sigma^{y}$.

$M \quad$ the set of absolute values on $\bar{K}$ extending those in $M_{K}$.

$\beta=2+\sqrt{3}$.

$E^{+}, E^{-} \in \operatorname{Div}(S) \otimes \mathbb{R}$, the divisors defined by

$$
E^{+}=\beta D_{m}^{x}-D_{n}^{y} \quad \text { and } \quad E^{-}=-D_{m}^{x}+\beta D_{n}^{y},
$$

where $m, n \in\{0,1,2\}$ are the indices fixed in $\S 1$. If it is necessary to specify $m$ and $n$, we will write $E_{m n}^{+}$and $E_{m n}^{-}$.

$\eta^{+}, \eta^{-} \in \operatorname{Pic}(S) \otimes \mathbb{R}$, the divisor classes of $E^{+}$and $E^{-}$respectively.

$h_{\eta^{+}}, h_{\eta^{-}}$Weil height functions $h_{\eta^{+}}, h_{\eta^{-}}: S(\bar{K}) \longrightarrow \mathbb{R}$ corresponding to the divisor classes $\eta^{+}$and $\eta^{-}$.

The divisor classes $\eta^{+}$and $\eta^{-}$are eigenclasses for the morphisms $\phi$ and $\psi$ respectively, each with eigenvalue $\beta^{2}>1$,

$$
\phi^{*} \eta^{+}=\beta^{2} \eta^{+} \quad \text { and } \quad \psi^{*} \eta^{-}=\beta^{2} \eta^{-} .
$$

Using these divisor class relations, Silverman [6] constructed two canonical heights $\hat{h}^{+}$and $\hat{h}^{-}$on $S$ as described in the following result. 
Theorem 3.1. There is a unique pair of functions

$$
\hat{h}^{+}, \hat{h}^{-}: S(\bar{K}) \longrightarrow \mathbb{R}
$$

with the following two properties:

(i) $\hat{h}^{ \pm}=h_{\eta^{ \pm}}+O(1)$.

(ii) $\hat{h}^{ \pm} \circ \sigma^{x}=\beta^{\mp 1} \hat{h}^{\mp} \quad$ and $\quad \hat{h}^{ \pm} \circ \sigma^{y}=\beta^{ \pm 1} \hat{h}^{\mp}$.

In addition, $\hat{h}^{+}$and $\hat{h}^{-}$also satisfy:

(iii) $\hat{h}^{+} \circ \phi=\beta^{2} \hat{h}^{+}$and $\hat{h}^{-} \circ \psi=\beta^{2} \hat{h}^{-}$.

(iv) For all $P \in S(\bar{K})$,

$$
\hat{h}^{+}(P)=\lim _{n \rightarrow \infty} \frac{h_{\eta^{+}}\left(\phi^{n} P\right)}{\beta^{2 n}} \quad \text { and } \quad \hat{h}^{-}(P)=\lim _{n \rightarrow \infty} \frac{h_{\eta^{-}}\left(\psi^{n} P\right)}{\beta^{2 n}} .
$$

Proof. Parts (i)-(iii) of this result correspond to the same parts of [6, Theorem 1.1]. Notice that (iii) is a consequence of (ii). The limit formulas given in (iv) may be proved using Tate's method, see [6, §3] or [2, Proposition 1.2].

It follows from [2, Theorem 1.1] that properties (i) and (iii) in Theorem 3.1 characterize the unique canonical heights $\hat{h}^{+}=\hat{h}_{S, \eta^{+}, \phi}$ and $\hat{h}^{-}=\hat{h}_{S, \eta^{-}, \psi}$ on $S$ associated with the divisor classes $\eta^{ \pm}$and the morphisms $\phi$ and $\psi$. To compute these canonical heights, we will decompose them into the canonical local heights constructed in [2]. We begin by refining the divisor class relations (13).

Lemma 3.2. Define functions $f^{x}, f^{y} \in K(S)$ by

$$
f^{x}(P)=G_{n}^{x}(\mathbf{x}) / x_{m}^{4} \quad \text { and } \quad f^{y}(P)=G_{m}^{y}(\mathbf{y}) / y_{n}^{4}, \quad \text { where } P=(\mathbf{x}, \mathbf{y}) \in S .
$$

$$
\begin{aligned}
& \left(\sigma^{x}\right)^{*} E^{+}=\beta^{-1} E^{-}-\operatorname{div}\left(f^{x}\right), \quad\left(\sigma^{y}\right)^{*} E^{-}=\beta^{-1} E^{+}-\operatorname{div}\left(f^{y}\right), \\
& \left(\sigma^{x}\right)^{*} E^{-}=\beta E^{+}+\beta \operatorname{div}\left(f^{x}\right), \quad\left(\sigma^{y}\right)^{*} E^{+}=\beta E^{-}+\beta \operatorname{div}\left(f^{y}\right) . \\
& \phi^{*} E^{+}=\beta^{2} E^{+}+\beta \operatorname{div}\left(f^{y} \circ \sigma^{x}\right)+\beta^{2} \operatorname{div}\left(f^{x}\right) \text {, } \\
& \psi^{*} E^{-}=\beta^{2} E^{-}+\beta \operatorname{div}\left(f^{x} \circ \sigma^{y}\right)+\beta^{2} \operatorname{div}\left(f^{y}\right) .
\end{aligned}
$$

Proof. (a) Clearly, $\left(\sigma^{x}\right)^{*} D_{m}^{x}=D_{m}^{x}$ and $\left(\sigma^{y}\right)^{*} D_{n}^{y}=D_{n}^{y}$. Further, the definition of $\sigma^{x}$ says that $(P)+\left(\sigma^{x} P\right)=p_{1}^{*}\left(p_{1} P\right)$ as zero cycles on $S$, so $\left(\sigma^{x}\right)^{*} D_{n}^{y}=$ $p_{1}^{*} p_{1 *}\left(D_{n}^{y}\right)-D_{n}^{y}$. Let $i, j$ be the indices so that $\{i, j, n\}=\{0,1,2\}$. Then

$$
\begin{aligned}
p_{1 *}\left(D_{n}^{y}\right) & =p_{1 *} \circ p_{2}^{*}\left(\left\{y_{n}=0\right\}\right) \\
& =\left\{\mathbf{x} \in \mathbb{P}^{2}: L(\mathbf{x}, \mathbf{y})=Q(\mathbf{x}, \mathbf{y})=y_{n}=0 \text { for some } y_{i}, y_{j}\right\} .
\end{aligned}
$$

Setting $y_{n}=0$ and eliminating $y_{i}, y_{j}$ from the equations

$$
\begin{aligned}
L(\mathbf{x}, \mathbf{y}) & =L_{i}^{x}(\mathbf{x}) y_{i}+L_{j}^{x}(\mathbf{x}) y_{j}=0 \text { and } \\
Q(\mathbf{x}, \mathbf{y}) & =Q_{i i}^{x}(\mathbf{x}) y_{i}{ }^{2}+Q_{i j}^{x}(\mathbf{x}) y_{i} y_{j}+Q_{j j}^{x}(\mathbf{x}) y_{j}{ }^{2}=0
\end{aligned}
$$

yields

$$
\begin{aligned}
p_{1 *}\left(D_{n}^{y}\right) & =\left\{\mathbf{x} \in \mathbb{P}^{2}: Q_{i i}^{x}(\mathbf{x}) L_{j}^{x}(\mathbf{x})^{2}-Q_{i j}^{x}(\mathbf{x}) L_{i}^{x}(\mathbf{x}) L_{j}^{x}(\mathbf{x})+Q_{j j}^{x}(\mathbf{x}) L_{i}^{x}(\mathbf{x})^{2}=0\right\} \\
& =\left\{\mathbf{x} \in \mathbb{P}^{2}: G_{n}^{x}(\mathbf{x})=0\right\} .
\end{aligned}
$$


Hence,

$$
\left(\sigma^{x}\right)^{*} D_{n}^{y}=p_{1}^{*}\left(\left\{G_{n}^{x}(\mathbf{x})=0\right\}\right)-D_{n}^{y}=4 D_{m}^{x}-D_{n}^{y}+\operatorname{div}\left(f^{x}\right),
$$

where $f^{x}$ is the function $f^{x}(\mathbf{x}, \mathbf{y})=G_{n}^{x}(\mathbf{x}) / x_{m}^{4}$.

Now using (14) and the definition of $E^{+}$, we compute

$$
\begin{aligned}
\left(\sigma^{x}\right)^{*} E^{+} & =\left(\sigma^{x}\right)^{*}\left(\beta D_{m}^{x}-D_{n}^{y}\right)=\beta D_{m}^{x}-\left(4 D_{m}^{x}-D_{n}^{y}+\operatorname{div}\left(f^{x}\right)\right) \\
& =(\beta-4) D_{m}^{x}+D_{n}^{y}-\operatorname{div}\left(f^{x}\right)=\beta^{-1}\left(-D_{m}^{x}+\beta D_{n}^{y}\right)-\operatorname{div}\left(f^{x}\right) \\
& =\beta^{-1} E^{-}-\operatorname{div}\left(f^{x}\right) .
\end{aligned}
$$

This proves the first formula in (a). Next we apply $\left(\sigma^{x}\right)^{*}$ to both sides to obtain

$$
E^{+}=\beta^{-1}\left(\sigma^{x}\right)^{*} E^{-}-\operatorname{div}\left(f^{x} \circ \sigma^{x}\right)=\beta^{-1}\left(\sigma^{x}\right)^{*} E^{-}-\operatorname{div}\left(f^{x}\right) .
$$

This gives the desired formula for $\left(\sigma^{x}\right)^{*} E^{-}$. The formulas for $\left(\sigma^{y}\right)^{*} E^{ \pm}$are proven similarly.

(b) We compute

$$
\begin{aligned}
\phi^{*} E^{+}=\left(\sigma^{x}\right)^{*}\left(\left(\sigma^{y}\right)^{*} E^{+}\right) & =\left(\sigma^{x}\right)^{*}\left(\beta E^{-}+\beta \operatorname{div}\left(f^{y}\right)\right) \\
& =\beta\left(\beta E^{+}+\beta \operatorname{div}\left(f^{x}\right)\right)+\beta \operatorname{div}\left(f^{y} \circ \sigma^{x}\right) \\
& =\beta^{2} E^{+}+\beta^{2} \operatorname{div}\left(f^{x}\right)+\beta \operatorname{div}\left(f^{y} \circ \sigma^{x}\right) .
\end{aligned}
$$

This gives the first formula in (b), and the second formula is proven similarly.

The divisor relations in Lemma 3.2 yield corresponding canonical local height functions.

Theorem 3.3. There is a unique pair of functions

$$
\hat{\lambda}^{ \pm}:\left(S(\bar{K}) \backslash\left|E^{ \pm}\right|\right) \times M \longrightarrow \mathbb{R}
$$

which are Weil local height functions for the divisors $E^{ \pm}$and which satisfy

$$
\begin{aligned}
& \hat{\lambda}^{+}(\phi P, v)=\beta^{2} \hat{\lambda}^{+}(P, v)+\beta v\left(f^{y}\left(\sigma^{x} P\right)\right)+\beta^{2} v\left(f^{x}(P)\right), \\
& \hat{\lambda}^{-}(\psi P, v)=\beta^{2} \hat{\lambda}^{-}(P, v)+\beta v\left(f^{x}\left(\sigma^{y} P\right)\right)+\beta^{2} v\left(f^{y}(P)\right) .
\end{aligned}
$$

Proof. This follows directly from [2, Theorem 2.1(b)] and the divisor relations proven in Lemma 3.2(b).

The global canonical heights $\hat{h}^{ \pm}$can then be computed by summing the local canonical heights over all absolute values.

Theorem 3.4. Let $L / K$ be a finite extension. Then with notation as in Theorems 3.1 and 3.3 ,

$$
\hat{h}^{ \pm}(P)=\frac{1}{[L: K]} \sum_{v \in M_{L}}\left[L_{v}: K_{v}\right] \hat{\lambda}^{ \pm}(P, v) \quad \text { for all } P \in S(L) \backslash\left|E^{ \pm}\right| .
$$




\section{Formulas FOR LOCAL HEIGHTS}

In this section we will develop formulas for computing local heights. We begin by fixing Weil local heights on $S$ for the divisors $D_{m}^{x}$ and $D_{n}^{y}$. So for any point $P=(\mathbf{x}, \mathbf{y}) \in S$, we define

$$
\begin{aligned}
\lambda_{D_{m}^{x}}(P, v) & =v\left(x_{m}\right)-\min \left\{v\left(x_{0}\right), v\left(x_{1}\right), v\left(x_{2}\right)\right\}, \\
\lambda_{D_{n}^{y}}(P, v) & =v\left(y_{n}\right)-\min \left\{v\left(y_{0}\right), v\left(y_{1}\right), v\left(y_{2}\right)\right\} .
\end{aligned}
$$

We then use these to fix Weil local heights associated with $E^{+}$and $E^{-}$:

$$
\lambda_{E^{+}}=\beta \lambda_{D_{m}^{x}}-\lambda_{D_{n}^{y}}, \quad \lambda_{E^{-}}=-\lambda_{D_{m}^{x}}+\beta \lambda_{D_{n}^{y}} .
$$

The divisor relations described in Lemma 3.2 lead to local height relations, which prompts us to define the following three pairs of "error functions":

$$
\begin{aligned}
\delta^{x}(P, v) & =\lambda_{E^{+}}\left(\sigma^{x} P, v\right)-\beta^{-1} \lambda_{E^{-}}(P, v)+v\left(f^{x}(P)\right), \\
\delta^{y}(P, v) & =\lambda_{E^{-}}\left(\sigma^{y} P, v\right)-\beta^{-1} \lambda_{E^{+}}(P, v)+v\left(f^{y}(P)\right), \\
\gamma^{+}(P, v) & =\lambda_{E^{+}}(\phi P, v)-\beta^{2} \lambda_{E^{+}}(P, v)-\beta v\left(f^{y}\left(\sigma^{x} P\right)\right)-\beta^{2} v\left(f^{x}(P)\right), \\
\gamma^{-}(P, v) & =\lambda_{E^{-}}(\psi P, v)-\beta^{2} \lambda_{E^{-}}(P, v)-\beta v\left(f^{x}\left(\sigma^{y} P\right)\right)-\beta^{2} v\left(f^{y}(P)\right), \\
\hat{\gamma}^{+}(P, v) & =\hat{\lambda}^{+}(P, v)-\lambda_{E^{+}}(P, v), \\
\hat{\gamma}^{-}(P, v) & =\hat{\lambda}^{-}(P, v)-\lambda_{E^{-}}(P, v) .
\end{aligned}
$$

Next we give some basic properties of these functions.

Lemma 4.1. Let $(P, v) \in S(\bar{K}) \times M$.

(a) The functions $\delta^{x}, \delta^{y}, \gamma^{+}, \gamma^{-}, \hat{\gamma}^{+}, \hat{\gamma}^{-}$all extend to $M_{K}$-bounded, $M_{K}$-continuous functions on $S(\bar{K}) \times M$. (See [3] for basic definitions.)

(b) $\quad \delta^{x}\left(\sigma^{x} P, v\right)=\delta^{x}(P, v) \quad$ and $\quad \delta^{y}\left(\sigma^{y} P, v\right)=\delta^{y}(P, v)$.

(c) $\quad \gamma^{+}(P, v)=-\beta^{2} \delta^{x}(P, v)-\beta \delta^{y}\left(\sigma^{x} P, v\right)$, $\gamma^{-}(P, v)=-\beta^{2} \delta^{y}(P, v)-\beta \delta^{x}\left(\sigma^{y} P, v\right)$.

(d) $\quad \gamma^{+}(P, v)=\beta^{2} \hat{\gamma}^{+}(P, v)-\hat{\gamma}^{+}(\phi P, v)$, $\gamma^{-}(P, v)=\beta^{2} \hat{\gamma}^{-}(P, v)-\hat{\gamma}^{-}(\psi P, v)$.

Proof. (a) The divisor relations given in Lemma 3.2 and functoriality of local height functions immediately imply the desired result for $\delta^{x}, \delta^{y}, \gamma^{+}, \gamma^{-}$. And the same result holds for $\hat{\gamma}^{+}, \hat{\gamma}^{-}$since Theorem 3.3 says that $\lambda_{E^{ \pm}}$and $\hat{\lambda}^{ \pm}$are Weil local height functions associated with the same divisors.

(b) For $(\mathbf{t}, v) \in \mathbb{P}^{2}(\bar{K}) \times M$, let $\lambda_{r}(\mathbf{t}, v)=\max _{i}\left\{v\left(t_{r} / t_{i}\right)\right\}$. As in Corollary 1.5, we write $P=(\mathbf{x}, \mathbf{y})$ and $\sigma^{x}(P)=\left(\mathbf{x}, \mathbf{y}^{\prime}\right)$. Notice that with this notation we have

$$
\lambda_{D_{n}^{y}}(P, v)=\lambda_{n}(\mathbf{y}, v) \quad \text { and } \quad \lambda_{D_{n}^{y}}\left(\sigma^{x} P, v\right)=\lambda_{n}\left(\mathbf{y}^{\prime}, v\right) .
$$

Expanding (18) using (15) and (17), we compute

$$
\begin{aligned}
\delta^{x}(P, v) & =\beta \lambda_{D_{m}^{x}}\left(\sigma^{x} P, v\right)-\lambda_{D_{n}^{y}}\left(\sigma^{x} P, v\right)+\beta^{-1} \lambda_{D_{m}^{x}}(P, v)-\lambda_{D_{n}^{y}}(P, v)+v\left(f^{x}(P)\right) \\
& =4 \lambda_{m}(\mathbf{x}, v)-\lambda_{n}(\mathbf{y}, v)-\lambda_{n}\left(\mathbf{y}^{\prime}, v\right)+v\left(G_{n}^{x}(\mathbf{x})\right)-4 v\left(x_{m}\right) \\
& =v\left(G_{n}^{x}(\mathbf{x})\right)-4 \min _{0 \leq i \leq 2}\left\{v\left(x_{i}\right)\right\}-\max _{0 \leq i \leq 2}\left\{v\left(\frac{y_{n}}{y_{i}}\right)\right\}-\max _{0 \leq i \leq 2}\left\{v\left(\frac{y_{n}^{\prime}}{y_{i}^{\prime}}\right)\right\} \\
(24) \quad & =v\left(G_{n}^{x}(\mathbf{x})\right)-4 \min _{0 \leq i \leq 2}\left\{v\left(x_{i}\right)\right\}-\max _{0 \leq i, j \leq 2}\left\{v\left(\frac{y_{n} y_{n}^{\prime}}{y_{i} y_{j}^{\prime}}\right)\right\} .
\end{aligned}
$$


This last expression is symmetric in $\mathbf{y}$ and $\mathbf{y}^{\prime}$, which shows that $\delta^{x}$ has the same value at $P=(\mathbf{x}, \mathbf{y})$ and $\sigma^{x} P=\left(\mathbf{x}, \mathbf{y}^{\prime}\right)$. This proves the first part of $(\mathrm{b})$, and the second part is proven similarly.

(c) Let $(P, v) \in\left(S(\bar{K}) \backslash\left|E^{+}\right|\right) \times M$. From (18) we have

$$
\lambda_{E^{+}}(P, v)=\lambda_{E^{+}}\left(\sigma^{x} \sigma^{x} P, v\right)=\delta^{x}\left(\sigma^{x} P, v\right)+\beta^{-1} \lambda_{E^{-}}\left(\sigma^{x} P, v\right)-v\left(f^{x}\left(\sigma^{x} P\right)\right) .
$$

But $f^{x}\left(\sigma^{x} P\right)=f^{x}(P)$ from the definition of $f^{x}$ and $\delta^{x}\left(\sigma^{x} P, v\right)=\delta^{x}(P, v)$ from (b), so after a little algebra we obtain

$$
\lambda_{E^{-}}\left(\sigma^{x} P, v\right)=\beta \lambda_{E^{+}}(P, v)+\beta v\left(f^{x}(P)\right)-\beta \delta^{x}(P, v) .
$$

Similarly, it follows from (19) that

$$
\lambda_{E^{+}}\left(\sigma^{y} P, v\right)=\beta \lambda_{E^{-}}(P, v)+\beta v\left(f^{y}(P)\right)-\beta \delta^{y}(P, v) .
$$

Using (25) and (26), we obtain

$$
\begin{aligned}
& \lambda_{E^{+}}(\phi P, v)=\lambda_{E^{+}}\left(\sigma^{y} \sigma^{x} P, v\right)=\beta \lambda_{E^{-}}\left(\sigma^{x} P, v\right)+\beta v\left(f^{y}\left(\sigma^{x} P\right)\right)-\beta \delta^{y}\left(\sigma^{x} P, v\right) \\
& \quad=\beta\left(\beta \lambda_{E^{+}}(P, v)+\beta v\left(f^{x}(P)\right)-\beta \delta^{x}(P, v)\right)+\beta v\left(f^{y}\left(\sigma^{x} P\right)\right)-\beta \delta^{y}\left(\sigma^{x} P, v\right) .
\end{aligned}
$$

Comparing this last equation with (20), we conclude that

$$
\gamma^{+}(P, v)=-\beta^{2} \delta^{x}(P, v)-\beta \delta^{y}\left(\sigma^{x} P, v\right),
$$

for all $P \in S(\bar{K}) \backslash\left|E^{+}\right|$and all $v \in M$. But $\gamma^{+}, \delta^{x}$ and $\delta^{y}$ are $M$-continuous functions on $S \times M$, and $S \backslash\left|E^{+}\right|$is a Zariski open subset of $S$, so it follows from [3, Chapter 10, Proposition 1.5] that (27) holds for all $(P, v) \in S \times M$. This proves the first part of (c), and the other part is proven similarly.

(d) To ease notation, we will write

$$
V(P)=\beta v\left(f^{y}\left(\sigma^{x} P\right)\right)+\beta^{2} v\left(f^{x}(P)\right),
$$

so Theorem 3.3 and (20) have the compact form

$$
\hat{\lambda}^{+} \circ \phi=\beta^{2} \hat{\lambda}^{+}+V \quad \text { and } \quad \lambda_{E^{+}} \circ \phi=\beta^{2} \lambda_{E^{+}}+V+\gamma^{+} .
$$

Using (28) and the definition (22) of $\hat{\gamma}^{+}$, it is now a simple matter to compute

$$
\begin{aligned}
\beta^{2} \hat{\gamma}^{+}-\hat{\gamma}^{+} \circ \phi & =\beta^{2}\left(\hat{\lambda}^{+}-\lambda_{E^{+}}\right)-\left(\hat{\lambda}^{+} \circ \phi-\lambda_{E^{+}} \circ \phi\right) \\
& =\beta^{2}\left(\hat{\lambda}^{+}-\lambda_{E^{+}}\right)-\left(\left(\beta^{2} \hat{\lambda}^{+}+V\right)-\left(\beta^{2} \lambda_{E^{+}}+V+\gamma^{+}\right)\right) \\
& =\gamma^{+} .
\end{aligned}
$$

This proves the first identity in (d), and the second is proven similarly.

Our final task in this section is to use the functions $\delta^{x}, \delta^{y}, \gamma^{+}, \gamma^{-}$to give convergent series for the canonical local height functions. These series can, in principle, be used for computations, although we will later modify them to make them more practical. However, an important consequence of our result is that if the error functions are zero, then the naive Weil local height is already the canonical local height. In the next section we will give a sufficient condition involving good reduction for this to occur at a non-Archimedean absolute value. For a more thorough investigation of the connection between degenerate reduction and canonical local heights, see [1]. 
Proposition 4.2. We have

$$
\begin{aligned}
\hat{\lambda}^{+}(P, v) & =\lambda_{E^{+}}(P, v)+\sum_{n \geq 0} \beta^{-2 n-2} \gamma^{+}\left(\phi^{n} P, v\right) \\
& =\lambda_{E^{+}}(P, v)-\delta^{x}(P, v)-\sum_{n \geq 1} \beta^{-2 n}\left(\delta^{x}\left(\phi^{n} P, v\right)+\beta \delta^{y}\left(\phi^{n} P, v\right)\right), \\
\hat{\lambda}^{-}(P) & =\lambda_{E^{-}}(P, v)+\sum_{n \geq 0} \beta^{-2 n-2} \gamma^{-}\left(\psi^{n} P, v\right) \\
& =\lambda_{E^{-}}(P, v)-\delta^{y}(P, v)-\sum_{n \geq 1} \beta^{-2 n}\left(\delta^{y}\left(\psi^{n} P, v\right)+\beta \delta^{x}\left(\psi^{n} P, v\right)\right) .
\end{aligned}
$$

Proof. We use Lemma 4.1(d) to write

$$
\lambda_{E^{+}}+\sum_{n \geq 0} \beta^{-2 n-2} \gamma^{+} \circ \phi^{n}=\lambda_{E^{+}}+\sum_{n \geq 0} \beta^{-2 n-2}\left(\beta^{2} \hat{\gamma}^{+} \circ \phi^{n}-\hat{\gamma}^{+} \circ \phi^{n+1}\right) .
$$

We know that $\hat{\gamma}^{+}$is bounded on $S\left(K_{v}\right)$ from Lemma 4.1(a), and $\beta>1$, so we are allowed to rearrange the terms in the series. The terms telescope, so we find that

$$
\lambda_{E^{+}}+\sum_{n \geq 0} \beta^{-2 n-2} \gamma^{+} \circ \phi^{n}=\lambda_{E^{+}}+\hat{\gamma}^{+}=\hat{\lambda}^{+} .
$$

This proves the first formula for $\hat{\lambda}^{+}$.

To prove the second formula, we compute

$$
\begin{aligned}
\hat{\lambda}^{+} & =\lambda_{E^{+}}+\sum_{n \geq 0} \beta^{-2 n-2} \gamma^{+} \circ \phi^{n} \quad \text { from above, } \\
& =\lambda_{E^{+}}-\sum_{n \geq 0} \beta^{-2 n-2}\left(\beta^{2} \delta^{x} \circ \phi^{n}+\beta \delta^{y} \circ \sigma^{x} \circ \phi^{n}\right) \quad \text { from Lemma 4.1(c), } \\
& =\lambda_{E^{+}}-\sum_{n \geq 0} \beta^{-2 n-2}\left(\beta^{2} \delta^{x} \circ \phi^{n}+\beta \delta^{y} \circ \phi^{n+1}\right) \quad \text { from Lemma 4.1(b), } \\
& =\lambda_{E^{+}}-\delta^{x}-\sum_{n \geq 1} \beta^{-2 n}\left(\delta^{x} \circ \phi^{n}+\beta \delta^{y} \circ \phi^{n}\right) .
\end{aligned}
$$

This proves the second formula for $\hat{\lambda}^{+}$. The formulas for $\hat{\lambda}^{-}$are proven similarly.

The functions $\gamma^{+}, \gamma^{-}, \delta^{x}, \delta^{y}$ are bounded on $S\left(K_{v}\right)$ from Lemma 4.1(a), so the series in Proposition 4.2 converge quite rapidly. More precisely, using the first $N$ terms of the series gives an error of $O\left(\beta^{-2 N}\right)$, where the big- $O$ constant depends on the equations defining the surface $S$. The following corollary makes this remark more precise. We will see in $\S 8$ how to use this corollary for practical calculations.

Corollary 4.3. Let $v \in M_{K}$. Suppose that $C^{x}$ and $C^{y}$ are quantities so that

$$
\left|\delta^{x}(P, v)\right| \leq C^{x} \quad \text { and } \quad\left|\delta^{y}(P, v)\right| \leq C^{y} \quad \text { for all } P \in S(K) .
$$


(a) Let $Q \in S(K)$ be a point satisfying

$$
\delta^{x}\left(\phi^{n} Q, v\right)=\delta^{y}\left(\phi^{n} Q, v\right)=0 \quad \text { for all } 0 \leq n \leq N .
$$

Then

$$
\left|\hat{\gamma}^{+}(Q, v)\right|=\left|\hat{\lambda}^{+}(Q, v)-\lambda_{E^{+}}(Q, v)\right| \leq \frac{\beta^{-2 N}}{\beta^{2}-1}\left(C^{x}+\beta C^{y}\right) .
$$

(b) Let $Q \in S(K)$ be a point satisfying

$$
\delta^{x}\left(\psi^{n} Q, v\right)=\delta^{y}\left(\psi^{n} Q, v\right)=0 \quad \text { for all } 0 \leq n \leq N .
$$

Then

$$
\left|\hat{\gamma}^{-}(Q, v)\right|=\left|\hat{\lambda}^{-}(Q, v)-\lambda_{E^{-}}(Q, v)\right| \leq \frac{\beta^{-2 N}}{\beta^{2}-1}\left(C^{y}+\beta C^{x}\right) .
$$

Proof. By symmetry, it suffices to prove (a). To ease notation, we will omit $v$ from the notation. We compute

$$
\begin{aligned}
\mid \hat{\lambda}^{+}(Q) & -\lambda_{E^{+}}(Q) \mid \\
& \leq\left|\delta^{x}(Q)\right|+\sum_{n \geq 1} \beta^{-2 n}\left(\left|\delta^{x}\left(\phi^{n} Q\right)\right|+\beta\left|\delta^{y}\left(\phi^{n} Q\right)\right|\right), \quad \text { from Proposition 4.2, } \\
& =\sum_{n \geq N+1} \beta^{-2 n}\left(\left|\delta^{x}\left(\phi^{n} Q\right)\right|+\beta\left|\delta^{y}\left(\phi^{n} Q\right)\right|\right), \quad \text { from }(30), \\
& \leq \sum_{n \geq N+1} \beta^{-2 n}\left(C^{x}+\beta C^{y}\right), \quad \text { from }(29), \\
& =\beta^{-2 N}\left(C^{x}+\beta C^{y}\right) /\left(\beta^{2}-1\right) .
\end{aligned}
$$

\section{The CANONICAL LOCAL HEIGHT FOR NON-ARCHIMEDEAN $v$}

In this section we will give an explicit formula for the error functions $\delta^{x}, \delta^{y}$ for non-Archimedean absolute values.

Definition. Let $\mathbf{a} \in \mathbb{P}^{2}(\bar{K})$ and $v \in M$ be a non-Archimedean absolute value. We say that $\left[a_{0}, a_{1}, a_{2}\right]$ are $v$-minimal coordinates for $\mathbf{a}$ if $\min \left\{v\left(a_{0}\right), v\left(a_{1}\right), v\left(a_{2}\right)\right\}=0$.

Theorem 5.1. Let $v \in M$ be a non-Archimedean absolute value, let $P \in S(\bar{K})$, and choose $v$-minimal coordinates $(\mathbf{a}, \mathbf{b})$ for $P$. Then

$$
\delta^{x}(P, v)=\min \left\{v\left(G_{0}^{x}(\mathbf{a})\right), v\left(G_{1}^{x}(\mathbf{a})\right), v\left(G_{2}^{x}(\mathbf{a})\right), v\left(H_{01}^{x}(\mathbf{a})\right), v\left(H_{02}^{x}(\mathbf{a})\right), v\left(H_{12}^{x}(\mathbf{a})\right)\right\},
$$

$$
\delta^{y}(P, v)=\min \left\{v\left(G_{0}^{y}(\mathbf{b})\right), v\left(G_{1}^{y}(\mathbf{b})\right), v\left(G_{2}^{y}(\mathbf{b})\right), v\left(H_{01}^{y}(\mathbf{b})\right), v\left(H_{02}^{y}(\mathbf{b})\right), v\left(H_{12}^{y}(\mathbf{b})\right)\right\} .
$$

Proof. By hypothesis, $S$ has no degenerate fibers, so by Proposition 1.4 the six quartic forms $G_{i}^{x}(\mathbf{x}), H_{i j}^{x}(\mathbf{x})$ have no common zeros. It follows that both sides of (32) are $M_{K}$-bounded, $M_{K}$-continuous functions. It thus suffices to prove that 
they are equal on a Zariski open subset [3, Chapter 10, Proposition 1.5], so we may assume that $G_{i}^{x}(\mathbf{a}) \neq 0$ for all $0 \leq i \leq 2$.

Writing $\sigma^{x} P=\left(\mathbf{a}, \mathbf{b}^{\prime}\right)$, we recall from Corollary 1.5 that $\mathbf{b}$ and $\mathbf{b}^{\prime}$ satisfy

$$
\left[b_{i} b_{i}^{\prime}, b_{i} b_{j}^{\prime}+b_{i}^{\prime} b_{j}, b_{j} b_{j}^{\prime}\right]=\left[G_{i}^{x}(\mathbf{a}),-H_{i j}^{x}(\mathbf{a}), G_{j}^{x}(\mathbf{a})\right] .
$$

This is a relation in $\mathbb{P}^{2}$, so there is a nonzero constant $\mu$ such that

$$
G_{i}^{x}(\mathbf{a})=\mu b_{i} b_{i}^{\prime} \quad \text { and } \quad-H_{i j}^{x}(\mathbf{a})=\mu\left(b_{i} b_{j}^{\prime}+b_{i}^{\prime} b_{j}\right) \quad \text { for all } i, j .
$$

We evaluate the formula $(24)$ for $\delta^{x}$ at $P=(\mathbf{a}, \mathbf{b})$ to obtain

$$
\delta^{x}(P, v)=v\left(G_{n}^{x}(\mathbf{a})\right)-4 \min _{0 \leq i \leq 2}\left\{v\left(a_{i}\right)\right\}-\max _{0 \leq i, j \leq 2}\left\{v\left(b_{n} b_{n}^{\prime} / b_{i} b_{j}^{\prime}\right)\right\} .
$$

By (34) and the fact that a has $v$-minimal coordinates, this becomes

$$
\delta^{x}(P, v)=v(\mu)+\min _{0 \leq i, j \leq 2}\left\{v\left(b_{i} b_{j}^{\prime}\right)\right\} .
$$

In order to complete the proof of Theorem 5.1, we will use the following elementary result.

Lemma 5.2. Let $v \in M$ be non-Archimedean and let $x, x^{\prime}, y, y^{\prime} \in \bar{K}$. Then

$$
\min \left\{v\left(x x^{\prime}\right), v\left(x y^{\prime}+x^{\prime} y\right), v\left(y y^{\prime}\right)\right\}=\min \left\{v\left(x x^{\prime}\right), v\left(x y^{\prime}\right), v\left(x^{\prime} y\right), v\left(x^{\prime} y^{\prime}\right)\right\} .
$$

Proof. See [3, Chapter 3, Proposition 2.1] or [4, VIII.5.9].

Resuming the proof of Theorem 5.1, we apply Lemma 5.2 repeatedly to (35). More precisely, for any $0 \leq i \leq j \leq 2$ we have

$$
\begin{aligned}
\min \left\{v\left(b_{i} b_{i}^{\prime}\right), v\left(b_{i} b_{j}^{\prime}\right),\right. & \left.v\left(b_{i}^{\prime} b_{j}\right), v\left(b_{j} b_{j}^{\prime}\right)\right\} \\
& =\min \left\{v\left(b_{i} b_{i}^{\prime}\right), v\left(b_{i} b_{j}^{\prime}+b_{i}^{\prime} b_{j}\right), v\left(b_{j} b_{j}^{\prime}\right)\right\} \\
& =\min \left\{v\left(\mu^{-1} G_{i}^{x}(\mathbf{a})\right), v\left(-\mu^{-1} H_{i j}^{x}(\mathbf{a})\right), v\left(\mu^{-1} G_{j}^{x}(\mathbf{a})\right)\right\} .
\end{aligned}
$$

Taking the various values of $i, j$ and substituting into (35) gives the desired result.

An important corollary of Theorem 5.1 is an effective criterion which lets us easily calculate the canonical local height at all but finitely many absolute values.

Corollary 5.3. Let $v \in M$ be a non-Archimedean absolute value, and assume that the forms $L(\mathbf{x}, \mathbf{y}), Q(\mathbf{x}, \mathbf{y})$ defining $S$ have $v$-integral coordinates. Assume further that the six quartic forms $G_{i}^{x}(\mathbf{x}), H_{i j}^{x}(\mathbf{x})$ have no common roots in the residue field $\bar{k}_{v}$, and similarly that the six quartic forms $G_{i}^{y}(\mathbf{y}), H_{i j}^{y}(\mathbf{y})$ have no common roots in $\bar{k}_{v}$. Then the canonical local height functions on $S$ are given by

$$
\hat{\lambda}^{+}(P, v)=\lambda_{E^{+}}(P, v) \quad \text { and } \quad \hat{\lambda}^{-}(P, v)=\lambda_{E^{-}}(P, v) \text {, }
$$

where to evaluate $\lambda_{E^{+}}(P, v)$ and $\lambda_{E^{-}}(P, v)$, we write $P=(\mathbf{a}, \mathbf{b})$ using $v$-minimal coordinates.

Proof. The conditions we have imposed on the quartic forms combined with Theorem 5.1 imply that

$$
\delta^{x}(P, v)=\delta^{y}(P, v)=0 \quad \text { for all } P \in S(\bar{K}) .
$$

The desired result then follows immediately from Proposition 4.2. 


\section{An Algorithm to COMPute $\sigma^{x}, \sigma^{y}, \phi$ And $\psi$}

In this section we will describe an algorithm to compute the automorphsims $\sigma^{x}$, $\sigma^{y}, \phi$, and $\psi$ on the surface $S$. In view of the fact that $\phi=\sigma^{y} \circ \sigma^{x}$ and $\psi=\sigma^{x} \circ \sigma^{y}$, it suffices to compute $\sigma^{x}$ and $\sigma^{y}$. The following result performs this task.

Algorithm 6.1. Let $P=(\mathbf{a}, \mathbf{b}) \in S$, where we no longer assume that all fibers of $S$ are nondegenerate.

(a) Assume that $S_{\mathbf{a}}^{x}$ is a nondegenerate fiber, and write $\sigma^{x} P=\left(\mathbf{a}, \mathbf{b}^{\prime}\right)$. Then

$$
\mathbf{b}^{\prime}= \begin{cases}{\left[b_{0} G_{0}^{x}(\mathbf{a}),-b_{0} H_{01}^{x}(\mathbf{a})-b_{1} G_{0}^{x}(\mathbf{a}),-b_{0} H_{02}^{x}(\mathbf{a})-b_{2} G_{0}^{x}(\mathbf{a})\right]} & \text { if } b_{0} \neq 0, \\ {\left[-b_{1} H_{01}^{x}(\mathbf{a})-b_{0} G_{1}^{x}(\mathbf{a}), b_{1} G_{1}^{x}(\mathbf{a}),-b_{1} H_{12}^{x}(\mathbf{a})-b_{2} G_{1}^{x}(\mathbf{a})\right]} & \text { if } b_{1} \neq 0, \\ {\left[-b_{2} H_{02}^{x}(\mathbf{a})-b_{0} G_{2}^{x}(\mathbf{a}),-b_{2} H_{12}^{x}(\mathbf{a})-b_{1} G_{2}^{x}(\mathbf{a}), b_{2} G_{2}^{x}(\mathbf{a})\right]} & \text { if } b_{2} \neq 0 .\end{cases}
$$

(b) Assume that $S_{\mathbf{b}}^{y}$ is a nondegenerate fiber, and write $\sigma^{y} P=\left(\mathbf{a}^{\prime}, \mathbf{b}\right)$. Then

$$
\mathbf{a}^{\prime}= \begin{cases}{\left[a_{0} G_{0}^{y}(\mathbf{b}),-a_{0} H_{01}^{y}(\mathbf{b})-a_{1} G_{0}^{y}(\mathbf{b}),-a_{0} H_{02}^{y}(\mathbf{b})-a_{2} G_{0}^{y}(\mathbf{b})\right]} & \text { if } a_{0} \neq 0, \\ {\left[-a_{1} H_{01}^{y}(\mathbf{b})-a_{0} G_{1}^{y}(\mathbf{b}), a_{1} G_{1}^{y}(\mathbf{b}),-a_{1} H_{12}^{y}(\mathbf{b})-a_{2} G_{1}^{y}(\mathbf{b})\right]} & \text { if } a_{1} \neq 0, \\ {\left[-a_{2} H_{02}^{y}(\mathbf{b})-a_{0} G_{2}^{y}(\mathbf{b}),-a_{2} H_{12}^{y}(\mathbf{b})-a_{1} G_{2}^{y}(\mathbf{b}), a_{2} G_{2}^{y}(\mathbf{b})\right]} & \text { if } a_{2} \neq 0 .\end{cases}
$$

Proof. Let $(\mathbf{x}, \mathbf{y}) \in S$ be a generic point, and write $\sigma^{x}(\mathbf{x}, \mathbf{y})=\left(\mathbf{x}, \mathbf{y}^{\prime}\right)$. Corollary 1.5 tells us that

$$
y_{\ell} y_{\ell}^{\prime} / y_{0} y_{0}^{\prime}=G_{\ell}^{x}(\mathbf{x}) / G_{0}^{x}(\mathbf{x}) \quad \text { and } \quad G_{0}^{x}(\mathbf{x}) y_{\ell}^{\prime 2}+H_{0 \ell}^{x}(\mathbf{x}) y_{0}^{\prime} y_{\ell}^{\prime}+G_{\ell}^{x}(\mathbf{x}) y_{0}^{\prime 2}=0 .
$$

Substituting the first equation into the second allows us to eliminate $G_{\ell}^{x}(\mathbf{x})$, and then multiplying by $y_{0} / y_{\ell}^{\prime}$ gives

$$
G_{0}^{x}(\mathbf{x}) y_{0} y_{\ell}^{\prime}+H_{0 \ell}^{x}(\mathbf{x}) y_{0} y_{0}^{\prime}+G_{0}^{x}(\mathbf{x}) y_{0}^{\prime} y_{\ell}=0 .
$$

Applying (36) with $\ell=1$ and $\ell=2$ yields

$$
\begin{aligned}
\mathbf{y}^{\prime} & =\left[y_{0}^{\prime}, y_{1}^{\prime}, y_{2}^{\prime}\right]=\left[y_{0} y_{0}^{\prime} G_{0}^{x}(\mathbf{x}), y_{0} y_{1}^{\prime} G_{0}^{x}(\mathbf{x}), y_{0} y_{2}^{\prime} G_{0}^{x}(\mathbf{x})\right] \\
& =\left[y_{0} G_{0}^{x}(\mathbf{x}),-y_{0} H_{01}^{x}(\mathbf{x})-y_{1} G_{0}^{x}(\mathbf{x}),-y_{0} H_{02}^{x}(\mathbf{x})-y_{2} G_{0}^{x}(\mathbf{x})\right] .
\end{aligned}
$$

This gives the first part of (a), and performing a similar computation using $y_{1}$ and $y_{2}$ in place of $y_{0}$ yields the other two parts. Note, however, that this only shows that (a) is true for generic points on $S$.

In other words, we know that (a) is correct for the point $(\mathbf{a}, \mathbf{b})$ provided that the formulas in (a) do not give $[0,0,0]$. Suppose that $b_{0} \neq 0$, and suppose that the first formula in (a) gives $[0,0,0]$. We are going to show that the fiber $S_{\mathbf{a}}^{x}$ is degenerate, contrary to assumption. The fact that the first formula in (a) gives $[0,0,0]$ combined with our assumption that $b_{0} \neq 0$ means that

$$
G_{0}^{x}(\mathbf{a})=H_{01}^{x}(\mathbf{a})=H_{02}^{x}(\mathbf{a})=0 .
$$

The fiber $S_{\mathbf{a}}^{x}$ is the set of solutions to the three equations

$$
\begin{aligned}
& G_{0}^{x}(\mathbf{a}) Y_{1}^{2}+H_{01}^{x}(\mathbf{a}) Y_{0} Y_{1}+G_{1}^{x}(\mathbf{a}) Y_{0}^{2}=0, \\
& G_{0}^{x}(\mathbf{a}) Y_{2}^{2}+H_{02}^{x}(\mathbf{a}) Y_{0} Y_{2}+G_{2}^{x}(\mathbf{a}) Y_{0}^{2}=0, \\
& G_{1}^{x}(\mathbf{a}) Y_{2}^{2}+H_{12}^{x}(\mathbf{a}) Y_{1} Y_{2}+G_{2}^{x}(\mathbf{a}) Y_{1}^{2}=0 .
\end{aligned}
$$

(See Corollary 1.5.) Substituting (37) into these equations yields

$$
G_{1}^{x}(\mathbf{a}) Y_{0}^{2}=G_{2}^{x}(\mathbf{a}) Y_{0}^{2}=G_{1}^{x}(\mathbf{a}) Y_{2}^{2}+H_{12}^{x}(\mathbf{a}) Y_{1} Y_{2}+G_{2}^{x}(\mathbf{a}) Y_{1}^{2}=0,
$$

which shows that the solution set is (at least) one-dimensional. Hence $S_{\mathbf{a}}^{x}$ is degenerate. This completes the proof of (a), and the proof of (b) is similar. 


\section{An Algorithm TO COMPUte CANONiCAL LOCAL HEIGHTS}

In this section we are going to describe a series for the canonical local height $\hat{\lambda}^{+}=\hat{\lambda}_{S, E^{+}, \phi}$ associated with the divisor $E^{+}$and the automorphism $\phi=\sigma^{y} \circ$ $\sigma^{x}$. The reader will easily be able to reverse the roles of $\mathbf{x}$ and $\mathbf{y}$ to produce the analogous series for the canonical local height associated with the divisor $E^{-}$and automorphism $\psi=\phi^{-1}=\sigma^{x} \circ \sigma^{y}$. See the appendix for code implementing both algorithms.

We know from Lemma 3.2(b) that

$$
\phi^{*} E^{+}=\beta^{2} E^{+}+\operatorname{div}(f) \quad \text { with } \quad f=\left(\frac{G_{m}^{y}(\mathbf{y})}{y_{n}^{4}} \circ \sigma^{x}\right)^{\beta}\left(\frac{G_{n}^{x}(\mathbf{x})}{x_{m}^{4}}\right)^{\beta^{2}}
$$

For each pair of (possibly identical) indices $i, j \in\{0,1,2\}$ we define a rational function on $S$ by the formula

$$
t_{i j}=\frac{\left(x_{m} / x_{i}\right)^{\beta}}{\left(y_{n} / y_{j}\right)} \in K(S)^{*} \otimes \mathbb{R}
$$

We write the divisor of $t_{i j}$ as

$$
\operatorname{div}\left(t_{i j}\right)=E^{+}-D_{i j} \quad \text { and note that } \quad\left|D_{i j}\right|=\left\{x_{i}=0\right\} \cup\left\{y_{j}=0\right\}
$$

For example, $t_{m n}=1$ and $D_{m n}=E^{+}$.

The nine divisors $D_{i j}$ have empty intersection, so they form a set of parameters with which to calculate the convergent series for $\hat{\lambda}^{+}$described in [2]. As in [2], the next step is to define two additional rational functions

$$
w_{i j}=f \cdot t_{i j}^{\beta^{2}} \quad \text { and } \quad z_{i j}=\frac{f \cdot t_{i j}^{\beta^{2}}}{t_{i j} \circ \phi}
$$

with divisors

$$
\operatorname{div}\left(w_{i j}\right)=\phi^{*} E^{+}-\beta^{2} D_{i j} \quad \text { and } \quad \operatorname{div}\left(z_{i j}\right)=\phi^{*} D_{i j}-\beta^{2} D_{i j}
$$

Finally, for any set of four indices $i, j, k, l \in\{0,1,2\}$ we define the transition functions

$$
s_{i j k l}=\frac{z_{k l} w_{i j}}{w_{k l}}=\frac{f \cdot t_{i j}^{\beta^{2}}}{t_{k l} \circ \phi} \quad \text { with divisors } \quad \operatorname{div}\left(s_{i j k l}\right)=\phi^{*} D_{k l}-\beta^{2} D_{i j}
$$

The rational function $s_{i j k l}$ has poles and zeros contained in the support of $D_{i j}$ and $\phi^{*} D_{k l}$. Suppose that we want to evaluate $s_{i j k l}$ at a point $P$ which is not $v$ adically close to $\left|\operatorname{div}\left(s_{i j k l}\right)\right|$, which means that $\left|s_{i j k l}(P)\right|_{v}$ should not be large or close to 0 . If we attempt to evaluate $s_{i j k l}(P)$ by first calculating $f(P), t_{i j}(P)$ and $t_{k l}(\phi P)$ and then using formula (38), we are likely to run into trouble. The problem is that these three factors from (38) may individually be $v$-adically large or small. So we need to rewrite the formula for $s_{i j k l}$ to reflect any cancellation that occurs. 
To make the notation somewhat easier, we will write

$$
P=(\mathbf{x}, \mathbf{y}), \quad \sigma^{x} P=\left(\mathbf{x}, \mathbf{y}^{\prime}\right), \quad \phi P=\sigma^{y}\left(\mathbf{x}, \mathbf{y}^{\prime}\right)=\left(\mathbf{x}^{\prime \prime}, \mathbf{y}^{\prime}\right) .
$$

Then the definitions of $s_{i j k l}, t_{i j}$, and $f$ given above lead to the formula

$$
s_{i j k l}=\underbrace{\left(\frac{G_{m}^{y}\left(\mathbf{y}^{\prime}\right)}{\left(y_{n}^{\prime}\right)^{4}}\right)^{\beta}\left(\frac{G_{n}^{x}(\mathbf{x})}{x_{m}^{4}}\right)^{\beta^{2}}}_{f} \underbrace{\left(\frac{x_{m}}{x_{i}}\right)^{\beta^{3}}\left(\frac{y_{j}}{y_{n}}\right)^{\beta^{2}}}_{t_{i j}^{\beta^{2}}} \underbrace{\left(\frac{x_{k}^{\prime \prime}}{x_{m}^{\prime \prime}}\right)^{\beta}\left(\frac{y_{n}^{\prime}}{y_{l}^{\prime}}\right)}_{1 / t_{k l} \circ \phi} .
$$

Next we use the fact that $\beta^{2}=4 \beta-1$ to rewrite the exponent of $x_{m} / x_{i}$ as $\beta^{3}=$ $4 \beta^{2}-\beta$ and to rewrite the exponent of $y_{n}^{\prime} / y_{l}^{\prime}$ as $1=4 \beta-\beta^{2}$. Then a little algebra gives

$$
s_{i j k l}=\left(\frac{G_{m}^{y}\left(\mathbf{y}^{\prime}\right)}{\left(y_{l}^{\prime}\right)^{4}} \cdot \frac{x_{i} x_{k}^{\prime \prime}}{x_{m} x_{m}^{\prime \prime}}\right)^{\beta}\left(\frac{G_{n}^{x}(\mathbf{x})}{x_{i}^{4}} \cdot \frac{y_{j} y_{l}^{\prime}}{y_{n} y_{n}^{\prime}}\right)^{\beta^{2}} .
$$

The formula (39) is still not usable, since for example it gives a zero in the denominator if any of $x_{m}, y_{n}, y_{n}^{\prime}, x_{m}^{\prime \prime}$ is zero. In order to create a usable formula, we need to briefly recall the series for $\hat{\lambda}^{+}$described in [2]. To compute $\hat{\lambda}^{+}(Q, v)$ we take the sequence of translates $Q, \phi Q, \phi^{2} Q, \ldots$ and perform certain computations. Suppose that we have just performed the computation associated with $P=\phi^{n} Q$. In particular, we will have chosen indices $\left(i^{\prime}, j^{\prime}, i, j\right)$ so that at the previous stage we computed $s_{i^{\prime} j^{\prime} i j}\left(\phi^{n-1} Q\right)$. It is not important to know now how the value of $j$ was chosen, but we will see that $i$ can be chosen to satisfy

$$
\max \left\{\left|x_{0} / x_{i}\right|_{v},\left|x_{1} / x_{i}\right|_{v},\left|x_{2} / x_{i}\right|_{v}\right\}=1 .
$$

Next we compute the point $\phi P=\left(\mathbf{x}^{\prime \prime}, \mathbf{y}^{\prime}\right)$ and use the result to choose indices $(k, l)$ satisfying the conditions

$$
\begin{gathered}
\max \left\{\left|x_{0}^{\prime \prime} / x_{k}^{\prime \prime}\right|_{v},\left|x_{1}^{\prime \prime} / x_{k}^{\prime \prime}\right|_{v},\left|x_{2}^{\prime \prime} / x_{k}^{\prime \prime}\right|_{v}\right\}=1 \quad \text { and } \\
\max \left\{\left|y_{0}^{\prime} / y_{l}^{\prime}\right|_{v},\left|y_{1}^{\prime} / y_{l}^{\prime}\right|_{v},\left|y_{2}^{\prime} / y_{l}^{\prime}\right|_{v}\right\}=1 .
\end{gathered}
$$

Notice that the index $k$ is chosen so that it can become our $i$ when we replace $P$ by $\phi P$. The next term in the series for $\hat{\lambda}^{+}(Q)$ will be

$$
\beta^{-2 e} \log \left|s_{i j k l}\left(\phi^{e} Q\right)\right|_{v},
$$

and our general theory tells us that this term is bounded by $O\left(\beta^{-2 e}\right)$. Our task is to find the value of $s_{i j k l}\left(\phi^{e} Q\right)=s_{i j k l}(P)$ without dealing with numbers that are very large or very close to 0 . The formula (39) for $s_{i j k l}$ has two factors which we deal with separately, so we will write

$$
s_{i j k l}=A_{i k l}^{\beta} B_{i j l}^{\beta^{2}} \quad \text { with } \quad A_{i k l}=\frac{G_{m}^{y}\left(\mathbf{y}^{\prime}\right)}{\left(y_{l}^{\prime}\right)^{4}} \cdot \frac{x_{i} x_{k}^{\prime \prime}}{x_{m} x_{m}^{\prime \prime}} \quad \text { and } \quad B_{i j l}=\frac{G_{n}^{x}(\mathbf{x})}{x_{i}^{4}} \cdot \frac{y_{j} y_{l}^{\prime}}{y_{n} y_{n}^{\prime}} .
$$

We begin by calculating $B_{i j l}$. We consider two cases: 
Case 1B. $\left|y_{j} / y_{l}\right|_{v} \leq 1$.

The formula for $\sigma^{x}$ given in Corollary 1.5(a) tells us in particular that

$$
y_{l} y_{l}^{\prime} / y_{n} y_{n}^{\prime}=G_{l}^{x}(\mathbf{x}) / G_{n}^{x}(\mathbf{x}) .
$$

Hence,

$$
B_{i j l}=\frac{G_{n}^{x}(\mathbf{x})}{x_{i}^{4}} \cdot \frac{y_{l} y_{l}^{\prime}}{y_{n} y_{n}^{\prime}} \cdot \frac{y_{j}}{y_{l}}=\frac{G_{l}^{x}(\mathbf{x})}{x_{i}^{4}} \cdot \frac{y_{j}}{y_{l}} .
$$

Note that it is easy to compute $G_{l}^{x}(\mathbf{x}) / x_{i}^{4}$, since this number is a polynomial in the quantities $x_{0} / x_{i}, x_{1} / x_{i}$, and $x_{2} / x_{i}$, each of which has absolute value at most 1 by (40). Further, our assumption for Case 1 B says that $\left|y_{j} / y_{l}\right|_{v} \leq 1$, so the other factor of $B_{i j l}$ in (42) also has bounded absolute value. Thus (42) gives a good formula for computing $B_{i j l}$ in Case 1B.

Case 2B. $\left|y_{j} / y_{l}\right|_{v}>1$.

Corollary 1.5(a) tells us that the point $\mathbf{y} \in \mathbb{P}^{2}$ satisfies the homogeneous equation

$$
G_{j}^{x}(\mathbf{x}) y_{l}^{2}+H_{j l}^{x}(\mathbf{x}) y_{j} y_{l}+G_{l}^{x}(\mathbf{x}) y_{j}^{2}=0 .
$$

We divide this equation by $y_{j} y_{l}$ to obtain

$$
G_{j}^{x}(\mathbf{x})\left(y_{l} / y_{j}\right)+H_{j l}^{x}(\mathbf{x})+G_{l}^{x}(\mathbf{x})\left(y_{j} / y_{l}\right)=0 .
$$

Now we substitute this into the formula (42) for $B_{i j l}$, which yields

$$
B_{i j l}=-\frac{G_{j}^{x}(\mathbf{x})}{x_{i}^{4}} \cdot \frac{y_{l}}{y_{j}}-\frac{H_{j l}^{x}(\mathbf{x})}{x_{i}^{4}} .
$$

Each of the quantities

$$
\left|G_{j}^{x}(\mathbf{x}) / x_{i}^{4}\right|_{v}, \quad\left|y_{l} / y_{j}\right|_{v}, \quad\left|H_{j l}^{x}(\mathbf{x}) / x_{i}^{4}\right|_{v}
$$

in (43) is bounded above. Hence (43) gives a good formula to compute $B_{i j l}$ in Case 2B.

The computation of $A_{i k l}$ is very similar, the point being that (41) says the coordinate $y_{l}^{\prime}$ appearing in the denominator of $A_{i k l}$ is the largest coordinate of $\mathbf{y}^{\prime}$. We again consider two cases.

Case 1A. $\left|x_{i} / x_{k}\right|_{v} \leq 1$.

The formula for $\sigma^{y}$ in Corollary 1.5(b) tells us that

$$
x_{k} x_{k}^{\prime \prime} / x_{m} x_{m}^{\prime \prime}=G_{k}^{y}\left(\mathbf{y}^{\prime}\right) / G_{m}^{y}\left(\mathbf{y}^{\prime}\right) .
$$

Note that we are applying the formula for $\sigma^{y}$ to the point $\left(\mathbf{x}, \mathbf{y}^{\prime}\right)$. Hence,

$$
A_{i k l}=\frac{G_{m}^{y}\left(\mathbf{y}^{\prime}\right)}{\left(y_{l}^{\prime}\right)^{4}} \cdot \frac{x_{k} x_{k}^{\prime \prime}}{x_{m} x_{m}^{\prime \prime}} \cdot \frac{x_{i}}{x_{k}}=\frac{G_{k}^{y}\left(\mathbf{y}^{\prime}\right)}{\left(y_{l}^{\prime}\right)^{4}} \cdot \frac{x_{i}}{x_{k}} .
$$

Each of the two factors on the right is bounded, so (44) is a good formula for computing $A_{i k l}$ in Case 1A. 
Case 2A. $\left|x_{i} / x_{k}\right|_{v}>1$.

The point $\left(\mathbf{x}, \mathbf{y}^{\prime}\right)$ is a point of $S$, so Corollary 1.5(b) says that $\mathbf{x} \in \mathbb{P}^{2}$ satisfies

$$
G_{i}^{y}\left(\mathbf{y}^{\prime}\right) x_{k}^{2}+H_{i k}^{y}\left(\mathbf{y}^{\prime}\right) x_{i} x_{k}+G_{k}^{y}\left(\mathbf{y}^{\prime}\right) x_{i}^{2}=0 .
$$

Dividing this by $x_{i} x_{k}$ and substituting into the formula (44) for $A_{i k l}$ gives

$$
A_{i k l}=-\frac{G_{i}^{y}\left(\mathbf{y}^{\prime}\right)}{\left(y_{l}^{\prime}\right)^{4}} \cdot \frac{x_{k}}{x_{i}}-\frac{H_{i k}^{y}\left(\mathbf{y}^{\prime}\right)}{\left(y_{l}^{\prime}\right)^{4}}
$$

As before, each fraction in this expression for $A_{i k l}$ is bounded, so (45) can be used to compute $A_{i k l}$ in Case $2 \mathrm{~A}$.

We have now given a method for computing $B_{i j l}$ and $A_{i k l}$ which does not involve using very large numbers. On the other hand, the product $s_{i j k l}=A_{i k l}^{\beta} B_{i j l}^{\beta^{2}}$ is uniformly bounded away from 0 , so neither $B_{i j l}$ nor $A_{i k l}$ will be too small. This completes the description of the algorithm to compute $\hat{\lambda}^{+}$. See the appendix for code implementing this algorithm and the analogous algorithm for $\hat{\lambda}^{-}$.

\section{A NUMERICAL EXAMPLE}

We will consider the surface $S / \mathbb{Q}$ already studied in [6]. This is the K3 surface defined by the forms

$$
\begin{aligned}
& L(\mathbf{x}, \mathbf{y})=x_{0} y_{0}+x_{1} y_{1}+x_{2} y_{2} \\
& \begin{array}{r}
Q(\mathbf{x}, \mathbf{y})=x_{0}^{2} y_{0}^{2}+3 x_{0} x_{1} y_{0}^{2}+x_{1}^{2} y_{0}^{2}+4 x_{0}^{2} y_{0} y_{1}+3 x_{0} x_{1} y_{0} y_{1}-2 x_{2}^{2} y_{0} y_{1}-x_{0}^{2} y_{1}^{2} \\
+2 x_{1}^{2} y_{1}^{2}-x_{0} x_{2} y_{1}^{2}-4 x_{1} x_{2} y_{1}^{2}+5 x_{0} x_{2} y_{0} y_{2}-4 x_{1} x_{2} y_{0} y_{2} \\
+7 x_{0}^{2} y_{1} y_{2}+4 x_{1}^{2} y_{1} y_{2}+x_{0} x_{1} y_{2}^{2}+3 x_{2}^{2} y_{2}^{2} .
\end{array}
\end{aligned}
$$

For later reference, we list the associated $G_{i}$ 's and $H_{i j}$ 's in Table 1 (next page). In this section we will work over $\mathbb{Q}$, and $p$ will always denote a prime in $\mathbb{Z}$.

Our first job is to find those $p$ 's for which the error functions $\delta^{x}(\cdot, p)$ and $\delta^{y}(\cdot, p)$ can be nonzero. Theorem 5.1 tells us that $\delta^{x}(\cdot, p)$ is nonzero if and only if the six polynomials

$$
\left\{G_{0}^{x}, G_{1}^{x}, G_{2}^{x}, H_{01}^{x}, H_{02}^{x}, H_{12}^{x}\right\}
$$

have a common zero modulo $p$. (We say "zero" for a zero with at least one nonzero coordinate, or equivalently a zero in $\mathbb{P}^{2}$.) Elimination theory says that there is a finite set of polynomials in the coefficients of the $G_{i}^{x}$ 's and $H_{i j}^{x}$ 's whose vanishing is equivalent to the existence of a common zero. (See, e.g., $[8, \S 16.5]$.) However, we will use ad hoc methods to get the result we want.

Our first observation is that any five of the polynomials in (46) have a common zero. More precisely,

$$
\begin{array}{ll}
G_{0}^{x}=G_{1}^{x}=G_{2}^{x}=H_{02}^{x}=H_{12}^{x}=0 & \text { at }([0,0,1],[1,0,0]) \in S, \text { and } \\
G_{0}^{x}=G_{1}^{x}=H_{01}^{x}=H_{02}^{x}=H_{12}^{x}=0 & \text { at }([0,1,0],[0,0,1]) \in S .
\end{array}
$$

So in order to find some necessary conditions for the polynomials (46) to have a common zero modulo $p$, we will use some linear combinations of the given six 
TABLE 1 . The $G$ and $H$ polynomials attached to the surface $S$

$\begin{aligned} & G_{0}^{x}=x_{0} x_{1}^{3}-7 x_{0}^{2} x_{1} x_{2}-4 x_{1}^{3} x_{2}-x_{0}^{2} x_{2}^{2}+5 x_{1}^{2} x_{2}^{2}-x_{0} x_{2}^{3}-4 x_{1} x_{2}^{3} \\ & G_{1}^{x}=x_{0}^{3} x_{1}-x_{0}^{2} x_{2}^{2}+7 x_{0} x_{1} x_{2}^{2}+x_{1}^{2} x_{2}^{2} \\ & G_{2}^{x}=-x_{0}^{4}-4 x_{0}^{3} x_{1}+3 x_{0} x_{1}^{3}+x_{1}^{4}-x_{0}^{3} x_{2}-4 x_{0}^{2} x_{1} x_{2}+2 x_{0} x_{1} x_{2}^{2} \\ & H_{01}^{x}=2 x_{0}^{2} x_{1}^{2}-7 x_{0}^{3} x_{2}-4 x_{0} x_{1}^{2} x_{2}+4 x_{0}^{2} x_{2}^{2}+4 x_{0} x_{1} x_{2}^{2}+4 x_{1}^{2} x_{2}^{2}-2 x_{2}^{4} \\ & H_{02}^{x}=-7 x_{0}^{3} x_{1}-4 x_{0} x_{1}^{3}-2 x_{0}^{3} x_{2}-4 x_{0}^{2} x_{1} x_{2}+6 x_{0} x_{1}^{2} x_{2}-4 x_{1}^{3} x_{2}-2 x_{0}^{2} x_{2}^{2} \\ & H_{12}^{x}=7 x_{0}^{4}+4 x_{0}^{2} x_{1}^{2}-4 x_{0}^{3} x_{2}-6 x_{2}^{2} x_{1} x_{2}+10 x_{0} x_{1}^{2} x_{2}^{3}+2 x_{1}^{3} x_{2}+2 x_{0} x_{2}^{3} \\ & G_{0}^{y}=-2 y_{0} y_{1}^{3}+4 y_{1}^{3} y_{2}+y_{0}^{2} y_{2}^{2}+4 y_{0} y_{1} y_{2}^{2}+5 y_{1}^{2} y_{2}^{2}+4 y_{1} y_{2}^{3} \\ & G_{1}^{y}=-2 y_{0}^{3} y_{1}+y_{0} y_{1}^{2} y_{2}-y_{0}^{2} y_{2}^{2}+4 y_{0} y_{1} y_{2}^{2}-y_{1}^{2} y_{2}^{2}+7 y_{1} y_{2}^{3} \\ & G_{2}^{y}=y_{0}^{4}-3 y_{0}^{3} y_{1}+4 y_{0} y_{1}^{3}-y_{1}^{4}+4 y_{0}^{2} y_{1} y_{2}+7 y_{1}^{3} y_{2}-y_{0} y_{1} y_{2}^{2} \\ & H_{01}^{y}=-4 y_{0}^{2} y_{1}^{2}+4 y_{0} y_{1}^{2} y_{2}+y_{1}^{3} y_{2}+7 y_{0}^{2} y_{2}^{2}+4 y_{0} y_{1} y_{2}^{2}+y_{2}^{4} \\ & H_{02}^{y}=4 y_{0} y_{1}^{3}-y_{1}^{4}+2 y_{0}^{3} y_{2}+y_{0}^{2} y_{1} y_{2}+6 y_{0} y_{1}^{2} y_{2}+8 y_{0} y_{1} y_{2}^{2}-y_{1} y_{2}^{3} \\ & H_{12}^{y}=-4 y_{0}^{2} y_{1}^{2}+y_{0} y_{1}^{3}-7 y_{0}^{3} y_{2}-6 y_{0}^{2} y_{1} y_{2}+8 y_{0} y_{1}^{2} y_{2}-2 y_{1}^{3} y_{2}+14 y_{1}^{2} y_{2}^{2}-y_{0} y_{2}^{3} \\ &\end{aligned}$

polynomials. We will begin with the three polynomials

$$
G_{0}^{x}, \quad H_{01}^{x}+G_{1}^{x}, \quad G_{2}^{x} .
$$

If we take the polynomials $H_{01}^{x}+G_{1}^{x}$ and $G_{2}^{x}$ and set $x_{0}=0$, we get $5 x_{1}^{2} x_{2}^{2}-2 x_{2}^{4}$ and $x_{1}^{4}$. It follows that these two polynomials have no common zeros with $x_{0}=0$ except possibly in characteristic 2 . In the following we will use tildes to denote polynomials dehomogenized by setting $x_{0}=1$ or $y_{0}=1$ as appropriate. Thus,

$$
\tilde{G}_{0}^{x}\left(x_{1}, x_{2}\right)=G_{0}^{x}\left(1, x_{1}, x_{2}\right), \quad \ldots, \quad \tilde{H}_{12}^{y}\left(y_{1}, y_{2}\right)=H_{12}^{y}\left(1, y_{1}, y_{2}\right) .
$$

We begin by eliminating the variable $x_{1}$ from three polynomials $\tilde{G}_{0}^{x}, \tilde{G}_{1}^{x}+\tilde{H}_{01}^{x}$, and $\tilde{G}_{2}^{x}$. To do this we compute the two resultants

$$
\begin{aligned}
R_{1}^{x}= & \operatorname{Res}_{x_{1}}\left(\tilde{G}_{1}^{x}+\tilde{H}_{01}^{x}, \tilde{G}_{2}^{x}\right) \\
= & -11-535 x_{2}+5917 x_{2}^{2}-21158 x_{2}^{3}+26697 x_{2}^{4}+19555 x_{2}^{5}-100440 x_{2}^{6} \\
& +23041 x_{2}^{7}+58460 x_{2}^{8}-101581 x_{2}^{9}+41839 x_{2}^{10}+3744 x_{2}^{11}-15916 x_{2}^{12} \\
& +7336 x_{2}^{13}-1072 x_{2}^{14}+16 x_{2}^{16}, \\
R_{2}^{x}= & \operatorname{Res}_{x_{1}}\left(\tilde{G}_{0}^{x}, \tilde{G}_{1}^{x}+\tilde{H}_{01}^{x}\right) \\
= & 50 x_{2}^{2}-549 x_{2}^{3}+191 x_{2}^{4}-5603 x_{2}^{5}+1111 x_{2}^{6}-19186 x_{2}^{7}+2334 x_{2}^{8}-30256 x_{2}^{9} \\
& -1510 x_{2}^{10}-6657 x_{2}^{11}-7395 x_{2}^{12}-204 x_{2}^{13}-1068 x_{2}^{14} .
\end{aligned}
$$

Next we take the resultant of these two polynomials (with respect to the only remaining variable $x_{2}$ ) to arrive at the integer

$$
S_{1}^{x}=\operatorname{Res}_{x_{2}}\left(R_{1}^{x}, R_{2}^{x}\right)=614651210694951578069424669784292475173585750433
$$
05196295025171217169011687132016108827091702190420366131200. 
We now know that $\delta^{x}(\cdot, p)=0$ for all primes not dividing $S_{1}^{x}$.

It is easy to check that $S_{1}^{x}$ is divisible by $2^{16} 3^{16} 5^{2} 11^{2} 37 \cdot 43$, but there is no point in performing a complete integer factorization. Instead we perform the same calculation with the three new polynomials

$$
H_{12}^{x}, \quad H_{01}^{x}+G_{1}^{x}, \quad G_{2}^{x} .
$$

The second and third are the same as before, so have no common root with $x_{0}=0$. Again we dehomogenize and compute

$$
R_{3}^{x}=\operatorname{Res}_{x_{1}}\left(\tilde{H}_{01}^{x}+\tilde{G}_{1}^{x}, \tilde{G}_{2}^{x}\right) \quad \text { and } \quad R_{4}^{x}=\operatorname{Res}_{x_{1}}\left(\tilde{H}_{12}^{x}, \tilde{H}_{01}^{x}+\tilde{G}_{1}^{x}\right),
$$

and then the resultant of $R_{3}^{x}$ and $R_{4}^{x}$ with respect to $x_{2}$ is

$$
\begin{array}{r}
S_{2}^{x}=9681750560643217568603549778745296289233120916312184763882 \\
763457823823874559309071500584839679064448856568627200 .
\end{array}
$$

Again we can find some small factors of $S_{2}^{x}$, such as $2^{20} 3^{16} 5^{2} 61 \cdot 71$. But the crucial fact is that if the error term $\delta^{x}(\cdot, p)$ is ever nonzero, then $p$ must divide both $S_{1}^{x}$ and $S_{2}^{x}$. It is now a simple matter to compute

$$
\begin{aligned}
S_{12}^{x}=\operatorname{gcd}\left(S_{1}^{x}, S_{2}^{x}\right) & =324661155228895023119937989836800 \\
& =2^{16} 3^{16} 5^{2} \cdot 317 \cdot 14521485737273461,
\end{aligned}
$$

so $\delta(\cdot, p)=0$ except possibly at the five primes dividing $S_{12}^{x}$.

But there is no need to stop here. Next we try the polynomials

$$
G_{0}^{x}+H_{01}^{x}, \quad G_{1}^{x}, \quad G_{2}^{x} .
$$

The procedure outlined above gives

$$
S_{3}^{x}=-1075829737901132846394168194849857103159139004416,
$$

and then we compute

$$
\begin{aligned}
S_{123}^{x} & =\operatorname{gcd}\left(S_{1}^{x}, S_{2}^{x}, S_{3}^{x}\right)=13745412929469382340087808 \\
& =2^{12} 3^{6} \cdot 317 \cdot 14521485737273461 .
\end{aligned}
$$

Taking several other triples of linear combinations of the six polynomials in (46) leads to the same four primes, so it is for these primes that the error $\delta^{x}$ might be nonzero. Further, Theorem 5.1 implies that this multi-resultant gives an upper bound for the error function, namely

$$
\delta^{x}(P, p) \leq v_{p}\left(S_{123}^{x}\right) .
$$

In order to compute canonical heights on $S$, we must also find out when the other error function $\delta^{y}$ can be nonzero. Without giving any details, we compute as above

$$
\begin{gathered}
R_{1}^{y}=\operatorname{Res}_{y_{1}}\left(\tilde{G}_{1}^{y}+\tilde{H}_{01}^{y}, \tilde{G}_{2}^{y}\right), \quad R_{2}^{y}=\operatorname{Res}_{y_{1}}\left(\tilde{G}_{0}^{y}, \tilde{G}_{1}^{y}+\tilde{H}_{01}^{y}\right), \\
R_{3}^{y}=\operatorname{Res}_{y_{1}}\left(\tilde{H}_{12}^{y}, \tilde{G}_{1}^{y}+\tilde{H}_{01}^{y}\right), \quad S_{1}^{y}=\operatorname{Res}_{y_{2}}\left(R_{1}^{y}, R_{2}^{y}\right), \quad S_{2}^{y}=\operatorname{Res}_{y_{2}}\left(R_{1}^{y}, R_{3}^{y}\right), \\
S_{12}^{y}=\operatorname{gcd}\left(S_{1}^{y}, S_{2}^{y}\right)=2^{27} 5^{6} \cdot 31 \cdot 507593 \cdot 2895545793631, \\
R_{4}^{y}=\operatorname{Res}_{y_{1}}\left(\tilde{G}_{0}^{y}+\tilde{H}_{01}^{y}, \tilde{G}_{1}^{y}\right), \quad R_{5}^{y}=\operatorname{Res}_{y_{1}}\left(\tilde{G}_{1}^{y}, \tilde{G}_{2}^{y}\right), \quad S_{3}^{y}=\operatorname{Res}_{y_{2}}\left(R_{4}^{y}, R_{5}^{y}\right), \\
S_{123}^{y}=\operatorname{gcd}\left(S_{1}^{y}, S_{2}^{y}, S_{3}^{y}\right)=2^{27} \cdot 507593 \cdot 2895545793631 .
\end{gathered}
$$

Thus, $\delta^{y}(\cdot, p)$ is zero except possibly at the three primes dividing $S_{123}^{y}$. 
We summarize the above discussion in the next proposition.

Proposition 8.1. Let $S / \mathbb{Q}$ be the $\mathrm{K} 3$ surface described at the beginning of this section, and let $\delta^{x}$ and $\delta^{y}$ be the error functions $(18,19)$ associated with $S$. Then for all number fields $K$, all non-Archimedean absolute values $v$ on $K$, and all points $P \in S(\bar{K})$,

$$
\begin{aligned}
& 0 \leq \delta^{x}(P, v) \leq v\left(2^{12} \cdot 3^{6} \cdot 317 \cdot 14521485737273461\right) \quad \text { and } \\
& 0 \leq \delta^{y}(P, v) \leq v\left(2^{27} \cdot 507593 \cdot 2895545793631\right)
\end{aligned}
$$

Proof. Since $G_{x}^{*}$ and $H_{i j}^{*}$ have integer coefficients, it follows from Theorem 5.1 that $\delta^{x}$ and $\delta^{y}$ take on nonnegative values. This gives the lower bounds, and the upper bounds are just a summary of the discussion given above.

Next we give some useful estimates for points in $S(\mathbb{Q})$.

Proposition 8.2. Let $S / \mathbb{Q}$ be the K3 surface described at the beginning of this section, and let $\delta^{x}$ and $\delta^{y}$ be the error functions $(18,19)$ associated with $S$. Further let $P=(\mathbf{x}, \mathbf{y}) \in S(\mathbb{Q})$.

(a) $\delta^{x}(P, 3)=0$.

(b) $\delta^{y}(P, 2)=0$.

(c) $\delta^{x}(P, 2)= \begin{cases}1 & \text { if } \mathbf{x} \equiv[0,0,1](\bmod 2) \\ 0 & \text { otherwise. }\end{cases}$

(d) If $\mathbf{x} \equiv[0,1,0](\bmod 2)$ or $\mathbf{x} \equiv[1,0,0](\bmod 2)$, then

$$
\delta^{x}\left(\phi^{n} P, 2\right)=0 \quad \text { for all } n \in \mathbb{Z} .
$$

Proof. (a) Note that if $\delta^{x}(P, 3)>0$, then the $\mathbf{x}$-coordinate of $P$ is a solution of the simultaneous congruences

$$
G_{0}^{x}(\mathbf{x}) \equiv G_{1}^{x}(\mathbf{x}) \equiv G_{2}^{x}(\mathbf{x}) \equiv H_{01}^{x}(\mathbf{x}) \equiv H_{02}^{x}(\mathbf{x}) \equiv H_{12}^{x}(\mathbf{x}) \equiv 0 \quad(\bmod 3) .
$$

However, it is a simple matter to evaluate these polynomials at the 26 points in $\mathbb{P}^{2}\left(\mathbb{F}_{3}\right)$ and verify that they have no common roots. Hence $\delta^{x}(P, 3)=0$ for all $P \in \mathbb{P}^{2}\left(\mathbb{Q}_{3}\right)$.

(b) Similarly, one can check that the six polynomials $\left\{G_{i}^{y}, H_{i j}^{y}\right\}$ do not all vanish at any of the seven points $\mathbf{y} \in \mathbb{P}^{2}\left(\mathbb{F}_{2}\right)$, so $\delta^{y}(P, 2)=0$ for all $P \in \mathbb{P}^{2}\left(\mathbb{Q}_{2}\right)$.

(c,d) We begin with a brief examination of the surface $S$ in characteristic 2 . Our first observation is that $S / \mathbb{F}_{2}$ is singular, or more precisely,

$$
P_{1}=([0,0,1],[1,0,0]) \in S\left(\mathbb{F}_{2}\right) \text { is a singular point of } S / \mathbb{F}_{2} \text {. }
$$

There are 11 points in $S\left(\mathbb{F}_{2}\right)$, which we label as follows:

$$
\begin{gathered}
P_{1}=([0,0,1],[1,0,0]), \quad P_{2}=([0,0,1],[0,1,0]), \quad P_{3}=([0,0,1],[1,1,0]), \\
P_{4}=([0,1,0],[0,0,1]), \quad P_{5}=([1,0,0],[0,0,1]), \quad P_{6}=([1,0,0],[0,1,1]), \\
P_{7}=([1,0,1],[0,1,0]), \quad P_{8}=([1,0,1],[1,1,1]), \quad P_{9}=([0,1,1],[1,1,1]), \\
P_{10}=([1,1,1],[1,1,0]) \quad P_{11}=([1,1,1],[1,0,1]) .
\end{gathered}
$$

Evaluating the $G_{i}$ 's and $H_{i j}$ 's at these points, we see that $\sigma^{x}$ is not well defined (in characteristic 2) at the three points $P_{1}, P_{2}, P_{3}$, but it is well defined at the other 
TABLE 2. The action of $\sigma^{x}$ and $\sigma^{y}$ on $S\left(\mathbb{F}_{2}\right)$

\begin{tabular}{ccc}
\hline$P$ & $\sigma^{x} P$ & $\sigma^{y} P$ \\
\hline$P_{1}$ & - & $P_{1}$ \\
$P_{2}$ & - & $P_{7}$ \\
$P_{3}$ & - & $P_{10}$ \\
$P_{4}$ & $P_{4}$ & $P_{5}$ \\
$P_{5}$ & $P_{6}$ & $P_{4}$ \\
$P_{6}$ & $P_{5}$ & $P_{6}$ \\
$P_{7}$ & $P_{8}$ & $P_{2}$ \\
$P_{8}$ & $P_{7}$ & $P_{9}$ \\
$P_{9}$ & $P_{9}$ & $P_{8}$ \\
$P_{10}$ & $P_{11}$ & $P_{3}$ \\
$P_{11}$ & $P_{10}$ & $P_{11}$ \\
\hline
\end{tabular}

eight points in $S\left(\mathbb{F}_{2}\right)$. Further, $\sigma^{y}$ is well defined at all points of $S\left(\mathbb{F}_{2}\right)$. The action of the involutions $\sigma^{x}, \sigma^{y}$ on $S\left(\mathbb{F}_{2}\right)$ is given in Table 2 .

Notice in particular the closed loop made up of the three points $\left\{P_{4}, P_{5}, P_{6}\right\}$. It follows that if $P \in S(\mathbb{Q})$ reduces to one of these points modulo 2 , then the same is true for every iterate $\phi^{n}(P)$. Hence for such a point, $\delta^{x}\left(\phi^{n} P, 2\right)=0$ for all $n \in \mathbb{Z}$, which completes the proof of $(d)$.

It remains to verify $(\mathrm{c})$. If $\mathbf{x} \not \equiv[0,0,1](\bmod 2)$, then one of the $G_{i}^{x}$ 's or $H_{i j}^{x}$ 's is nonzero modulo 2 , so $\delta^{x}(P, 2)=0$. On the other hand, if $\mathbf{x} \equiv[0,0,1](\bmod 2)$, then evaluating the $G_{i}^{x}$ 's and $H_{i j}^{x}$ 's at $P$, we see that

$$
\begin{aligned}
H_{01}^{x}(\mathbf{x}) & \equiv 2(\bmod 4), \quad G_{0}^{x}(\mathbf{x}) \equiv 0(\bmod 2), \quad \text { and } \\
G_{1}^{x}(\mathbf{x}) & \equiv G_{2}^{x}(\mathbf{x}) \equiv H_{02}^{x}(\mathbf{x}) \equiv H_{12}^{x}(\mathbf{x}) \equiv 0(\bmod 4)
\end{aligned}
$$

It follows from Theorem 5.1 that $\delta^{x}(P, 2)=1$.

We are now ready to compute the canonical height of some representative points in $S(\mathbb{Q})$. We will begin by calculating $\hat{h}^{+}(Q)$ for the point

$$
Q=([0,1,0],[0,0,1]) .
$$

Note that Proposition 8.1 combined with Proposition 4.2 says that

$$
\hat{\lambda}^{+}(Q, p)=\lambda_{E^{+}}(Q, p)
$$

except possibly at the "bad primes"

$$
\{2,3,317,507593,2895545793631,14521485737273461\} \text {. }
$$

Further, Proposition 8.2(a,b,d) tells us that

$$
\delta^{x}\left(\phi^{n} Q, 2\right)=\delta^{x}\left(\phi^{n} Q, 3\right)=\delta^{y}\left(\phi^{n} Q, 2\right)=0 \quad \text { for all } n \in \mathbb{Z},
$$

and Proposition 8.1 gives $\delta^{y}\left(\phi^{n} Q, 3\right)=0$, so another application of Proposition 4.2 yields

$$
\hat{\lambda}^{+}(Q, 2)=\lambda_{E^{+}}(Q, 2) \quad \text { and } \quad \hat{\lambda}^{+}(Q, 3)=\lambda_{E^{+}}(Q, 3) .
$$


TABLE 3. The iterates $\phi^{n} Q$ modulo 317

\begin{tabular}{cc}
\hline$n$ & $\phi^{n} Q(\bmod 317)$ \\
\hline 0 & $([0,1,0],[0,0,1])$ \\
1 & $([316,0,0],[0,310,316])$ \\
2 & $([257,27,128],[52,183,226])$ \\
3 & $([144,113,104],[291,57,160])$ \\
4 & $([271,207,107],[292,98,90])$ \\
5 & $([155,94,226],[258,250,210])$ \\
6 & $([79,245,43],[311,124,285])$ \\
7 & $([312,227,306],[289,184,294])$ \\
8 & $([72,185,83],[293,17,235])$ \\
9 & $([243,312,125],[265,168,227])$ \\
10 & $([308,162,54],[41,227,224])$ \\
11 & $([132,300,136],[297,256,126])$ \\
12 & $([106,160,49],[241,263,179])$ \\
13 & $([53,70,118],[286,218,301])$ \\
14 & $([72,105,142],[201,158,67])$ \\
15 & $([110,231,204],[136,105,117])$ \\
\hline
\end{tabular}

This takes care of two of the bad primes.

In order to estimate $\hat{\lambda}^{+}(Q, p)$ at the other bad primes, we want to use Corollary 4.3. This means we need to find bounds $C^{x}$ and $C^{y}$ as in (29) and an integer $N$ as in (30). The first part is easy, since Proposition 8.1 provides absolute upper bounds for $\delta^{x}(P, p)$ and $\delta^{y}(P, p)$.

For the second part, we observe that the Algorithm 6.1 for computing $\sigma^{x}$ and $\sigma^{y}$ fails precisely when the $G_{i}$ 's and $H_{i j}$ 's have a common root. So if we work "modulo $p^{\prime}$, Theorem 5.1 says that the algorithm fails exactly when $\delta^{x}$ or $\delta^{y}$ is nonzero. This means that if we start with $Q$, and if we can use our algorithm to compute $\phi^{n} Q(\bmod p)$ without encountering a point with a $[0,0,0]$ coordinate, then $\delta^{x}\left(\phi^{n} Q, p\right)=\delta^{y}\left(\phi^{n} Q, p\right)=0$. It is a simple matter to program the algorithm for $\phi$, and since we only need to work modulo $p$, the numbers don't become too large. (In other words, it would not be possible to compute say $\phi^{20}(Q)$ exactly in $S(\mathbb{Q})$, but it is quite feasible to compute it in $S\left(\mathbb{F}_{p}\right)$ for any moderate size $p$.)

For example, the iterates $\phi^{n} Q(\bmod 317)$ are listed in Table 3 . It follows from Table 3 that $\delta^{x}\left(\phi^{n} Q, 317\right)=0$ for all $0 \leq n \leq 15$, and we already know from Proposition 8.1 that $\delta^{y}\left(\phi^{n} Q, 317\right)=0$ for all $n$. So we can apply Corollary 4.3 with

$$
p=317, \quad C^{x}=v(p)=\log (317), \quad C^{y}=0, \quad N=15,
$$

to obtain the estimate

$$
\left|\hat{\gamma}^{+}(Q, 317)\right|=\left|\hat{\lambda}^{+}(Q, 317)-\lambda_{E^{+}}(Q, 317)\right| \leq \frac{\beta^{-30}}{\beta^{2}-1} \log (317) \approx 3 \cdot 10^{-18} .
$$

We can deal with the other bad primes in a similar manner. Thus, Table 4 allows us to apply Corollary 4.3 with

$$
p=507593, \quad C^{x}=0, \quad C^{y}=v(p)=\log (507593), \quad N=15 .
$$


TABLE 4. The iterates $\phi^{n} Q$ modulo 507593

\begin{tabular}{cc}
\hline$n$ & $\phi^{n} Q(\bmod 507593)$ \\
\hline 0 & $([0,1,0],[0,0,1])$ \\
1 & $([507592,0,0],[0,507586,507592])$ \\
2 & $([505948,344,505185],[14078,253785,419137])$ \\
3 & $([440714,104662,327579],[476070,436327,483230])$ \\
$\vdots$ & $\vdots$ \\
14 & $([308966,48587,503331],[141252,226154,476629])$ \\
15 & $([474867,299570,409761],[119433,10607,79029])$ \\
\hline
\end{tabular}

This gives the estimate

$$
\left|\hat{\gamma}^{+}(Q, p)\right|=\left|\hat{\lambda}^{+}(Q, p)-\lambda_{E^{+}}(Q, p)\right| \leq \frac{\beta^{-29}}{\beta^{2}-1} \log (p) \approx 2.6 \cdot 10^{-17} \quad \text { for } p=507593 .
$$

We will not bother listing the corresponding tables for the two larger bad primes, but will merely give the results

$$
\begin{aligned}
\left|\hat{\gamma}^{+}(Q, p)\right| & =\left|\hat{\lambda}^{+}(Q, p)-\lambda_{E^{+}}(Q, p)\right| \\
& \leq \begin{cases}5.75 \cdot 10^{-17} & \text { for } p=2895545793631, \\
2 \cdot 10^{-17} & \text { for } p=14521485737273461 .\end{cases}
\end{aligned}
$$

Summing over places of $\mathbb{Q}$, these computations show that

$$
\hat{h}^{+}(Q) \approx \hat{\lambda}^{+}(Q, \infty)+\sum_{p \text { finite }} \lambda_{E^{+}}(Q, p)
$$

with an error of at most $1.1 \cdot 10^{-16}$. It remains to compute the Archimedean contribution. Of course, this is only valid if we choose for $E^{+}$a divisor $E_{m n}^{+}$ whose support does not contain $Q$, which in this case means taking $E_{12}^{+}$. Then all of the $\lambda_{E^{+}}(Q, p)$ 's vanish, so $\hat{h}^{+}(Q) \approx \hat{\lambda}^{+}(Q, \infty)$. It only remains to implement the algorithm described in $\S 7$ (see also the appendix) and use it to compute this Archimedean height. We carried out this computation to obtain the estimate

$$
\hat{h}^{+}(Q) \approx 0.147576 \text {. }
$$

Further, since $\sigma^{x}(Q)=Q$, we can use Theorem 3.1(ii) to get the other height for free,

$$
\hat{h}^{-}(Q)=\beta \hat{h}^{+}\left(\sigma^{x} Q\right)=\beta \hat{h}^{+}(Q) \approx 0.55076 .
$$

We will conclude by computing the height of the point

$$
R=([0,0,1],[1,0,0]) \in S(\mathbb{Q}) .
$$

Most of the calculation goes exactly the same as the calculation for $Q$. In particular, we find that the contribution from the bad primes $p \geq 3$ is negligible, so

$$
\hat{h}^{+}(R)=\hat{\lambda}^{+}(R, \infty)+\hat{\lambda}^{+}(R, 2) .
$$


Further, the algorithm for the Archimedean height gives

$$
\hat{\lambda}^{+}(R, \infty) \approx 0.892307,
$$

so it remains to analyze $\hat{\lambda}^{+}(R, 2)$.

First note that

$$
G_{0}^{x}(R)=G_{1}^{x}(R)=G_{2}^{x}(R)=H_{02}^{x}(R)=H_{12}^{x}(R)=0, \quad H_{01}^{x}(R)=-2 .
$$

It follows from Theorem 5.1 that

$$
\delta^{x}(R, 2)=\log (2) .
$$

Further, if we use the formula for $\sigma^{x}$ given in Algorithm 6.1 but work in the finite field $\mathbb{F}_{2}$, then we find that $\sigma^{x}(R)=([0,0,1],[0,0,0])$. In other words, we do not get a well-defined point in $\mathbb{P}^{2}\left(\mathbb{F}_{2}\right)$. However, suppose instead that we work to a higher power of 2. For example, if we work modulo 4 we find that

$$
\sigma^{x}(R) \equiv([0,0,1],[0,2,0]) \quad(\bmod 4) .
$$

We can then cancel a factor of 2 from the $\mathbf{y}$-coordinate, but we must reduce the exponent of our congruence. Thus

$$
\sigma^{x}(R) \equiv([0,0,1],[0,1,0]) \quad(\bmod 2) .
$$

As we continue to successively apply $\sigma^{y}$ and $\sigma^{x}$, we may again run into points whose $\mathbf{x}$ - or $\mathbf{y}$-coordinate is $[0,0,0]$. So we will work modulo a higher power of 2 . Table 5 gives $\phi^{n} R$ in the range $0 \leq n \leq 20$, beginning modulo $2^{12}$ and finishing modulo $2^{4}$. The table also lists the values of $\delta^{x}(\cdot, 2)$ and $\delta^{y}(\cdot, 2)$, where we are taking the normalized valuation at 2 . Of course, we already know that $\delta^{y}(\cdot, 2)=0$ for all points in $S(\mathbb{Q})$, so the last column is no surprise.

One observation is that $\delta^{x}$ appears to be nonzero in a very regular pattern. More precisely, it appears that

$$
\delta^{x}\left(\phi^{n} R\right)= \begin{cases}0 & \text { if } n \equiv 1,2,3(\bmod 5) \\ 1 & \text { if } n \equiv 0,4(\bmod 5)\end{cases}
$$

This suggests the existence of a "weak Néron model" for $S$ over $\operatorname{Spec}\left(\mathbb{Z}_{2}\right)$, as described in $[2, \S 6]$.

If this pattern continues, we could compute $\hat{\lambda}^{+}(R, 2)$ exactly, but in any case, we can use the table of values for $\delta^{x}\left(\phi^{n} R, 2\right)$ to estimate the quantity

$$
\begin{aligned}
\hat{\lambda}^{+}(R, 2) & =\lambda_{E_{20}^{+}}(R, 2)-\delta^{x}(R, 2)-\sum_{n \geq 1} \beta^{-2 n}\left(\delta^{x}\left(\phi^{n} R, 2\right)+\beta \delta^{y}\left(\phi^{n}, 2\right)\right) \\
& =0-\log 2-\sum_{n \geq 1} \beta^{-2 n} \delta^{x}\left(\phi^{n} R, 2\right) \quad \text { since } \delta^{y}=0 \text { on } S\left(\mathbb{Q}_{2}\right) .
\end{aligned}
$$

The table gives

$$
\begin{aligned}
& \sum_{n=1}^{20} \beta^{-2 n} \\
& \delta^{x}\left(\phi^{n} R, 2\right) \\
& \quad=\left(\beta^{-8}+\beta^{-10}+\beta^{-18}+\beta^{-20}+\beta^{-28}+\beta^{-30}+\beta^{-38}+\beta^{-40}\right) \log 2 \\
& \quad \approx 0.00001974 .
\end{aligned}
$$


TABLE 5 . The iterates $\phi^{n} R$ modulo powers of 2

\begin{tabular}{ccccc}
\hline$n$ & $\phi^{n} R$ & $\left(\bmod 2^{e}\right)$ & $\delta^{x}$ & $\delta^{y}$ \\
\hline 0 & $([0,0,1],[1,0,0])$ & $\left(\bmod 2^{12}\right)$ & 1 & 0 \\
1 & $([1,0,2047],[0,1,0])$ & $\left(\bmod 2^{11}\right)$ & 0 & 0 \\
2 & $([528,1931,1533],[2039,2047,2039])$ & $\left(\bmod 2^{11}\right)$ & 0 & 0 \\
3 & $([1585,1252,1599],[619,287,983])$ & $\left(\bmod 2^{11}\right)$ & 0 & 0 \\
4 & $([1880,56,1881],[804,649,488])$ & $\left(\bmod 2^{11}\right)$ & 1 & 0 \\
5 & $([88,96,345],[105,864,552])$ & $\left(\bmod 2^{10}\right)$ & 1 & 0 \\
6 & $([257,172,343],[436,329,192])$ & $\left(\bmod 2^{9}\right)$ & 0 & 0 \\
7 & $([16,27,149],[379,343,471])$ & $\left(\bmod 2^{9}\right)$ & 0 & 0 \\
8 & $([353,504,135],[399,495,431])$ & $\left(\bmod 2^{9}\right)$ & 0 & 0 \\
9 & $([416,392,1],[192,65,120])$ & $\left(\bmod 2^{9}\right)$ & 1 & 0 \\
10 & $([160,24,33],[193,160,96])$ & $\left(\bmod 2^{8}\right)$ & 1 & 0 \\
11 & $([65,8,55],[32,65,104])$ & $\left(\bmod 2^{7}\right)$ & 0 & 0 \\
12 & $([16,67,125],[127,111,127])$ & $\left(\bmod 2^{7}\right)$ & 0 & 0 \\
13 & $([97,28,7],[3,111,63])$ & $\left(\bmod 2^{7}\right)$ & 0 & 0 \\
14 & $([24,48,73],[52,57,112])$ & $\left(\bmod 2^{7}\right)$ & 1 & 0 \\
15 & $([24,40,9],[25,32,40])$ & $\left(\bmod 2^{6}\right)$ & 1 & 0 \\
16 & $([17,20,15],[4,25,24])$ & $\left(\bmod 2^{5}\right)$ & 0 & 0 \\
17 & $([16,19,5],[19,23,31])$ & $\left(\bmod 2^{5}\right)$ & 0 & 0 \\
18 & $([1,16,15],[23,15,23])$ & $\left(\bmod 2^{5}\right)$ & 0 & 0 \\
19 & $([0,16,1],[0,1,16])$ & $\left(\bmod 2^{5}\right)$ & 1 & 0 \\
20 & $([0,0,1],[1,0,0])$ & $\left(\bmod 2^{4}\right)$ & 1 & 0 \\
\hline
\end{tabular}

Further, the estimate $\delta^{x}(\cdot, 2) \leq \log 2$ provided by Proposition 8.2(c) gives

$$
\sum_{n \geq 21} \beta^{-2 n} \delta^{x}\left(\phi^{n} R, 2\right) \leq \sum_{n \geq 21} \beta^{-2 n} \log 2=\frac{\beta^{-40}}{\beta^{2}-1} \log 2<10^{-24} .
$$

Adding these gives $\hat{\lambda}^{+}(R, 2) \approx-0.693167$, and combining this with the Archimedean height yields

$$
\hat{h}^{+}(R) \approx 0.199140 .
$$

Finally, we can use the fact that $\sigma^{y} R=R$ to compute the other height

$$
\hat{h}^{-}(R)=\hat{h}^{-}\left(\sigma^{y} R\right)=\beta^{-1} \hat{h}^{+}(R) \approx 0.053359 .
$$

\section{APPENDix. IMPLEMENTATION OF ALGORITHMS}

In this appendix we give code to implement the algorithms described in this paper. We take $S$ to be the surface in $\mathbb{P}^{2} \times \mathbb{P}^{2}$ described by the simultaneous equations

$$
L(\mathbf{x}, \mathbf{y})=\sum_{i, j=0}^{2} a_{i j} x_{i} y_{j}=0, \quad Q(\mathbf{x}, \mathbf{y})=\sum_{i, j, k, l=0}^{2} b_{i j k l} x_{i} x_{j} y_{k} y_{l}=0 .
$$


We assume that $S$ has no degenerate fibers. We further assume that there are routines available to evaluate the polynomials

$$
G_{0}^{x}, G_{1}^{x}, G_{2}^{x}, H_{01}^{x}, H_{02}^{x}, H_{12}^{x} \quad \text { and } \quad G_{0}^{y}, G_{1}^{y}, G_{2}^{y}, H_{01}^{y}, H_{02}^{y}, H_{12}^{y}
$$

described by the formulas (1) and (2) in $\S 1$.

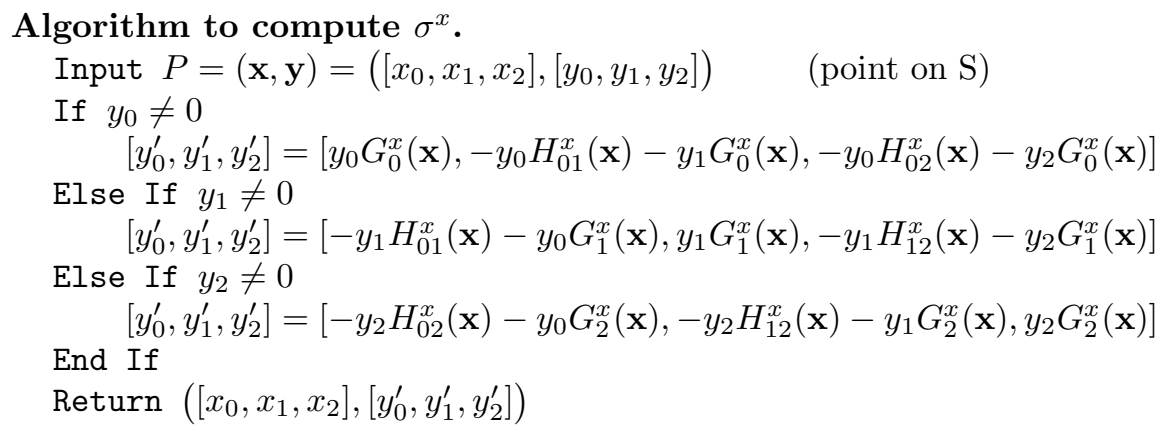

\section{Algorithm to compute $\sigma^{y}$.}

Input $P=(\mathbf{x}, \mathbf{y})=\left(\left[x_{0}, x_{1}, x_{2}\right],\left[y_{0}, y_{1}, y_{2}\right]\right) \quad$ (point on $\left.\mathrm{S}\right)$

If $x_{0} \neq 0$

$\left[x_{0}^{\prime}, x_{1}^{\prime}, x_{2}^{\prime}\right]=\left[x_{0} G_{0}^{y}(\mathbf{y}),-x_{0} H_{01}^{y}(\mathbf{y})-x_{1} G_{0}^{y}(\mathbf{y}),-x_{0} H_{02}^{y}(\mathbf{y})-x_{2} G_{0}^{y}(\mathbf{y})\right]$

Else If $x_{1} \neq 0$

$\left[x_{0}^{\prime}, x_{1}^{\prime}, x_{2}^{\prime}\right]=\left[-x_{1} H_{01}^{y}(\mathbf{y})-x_{0} G_{1}^{y}(\mathbf{y}), x_{1} G_{1}^{y}(\mathbf{y}),-x_{1} H_{12}^{y}(\mathbf{y})-x_{2} G_{1}^{y}(\mathbf{y})\right]$

Else If $x_{2} \neq 0$

End If

$$
\left[x_{0}^{\prime}, x_{1}^{\prime}, x_{2}^{\prime}\right]=\left[-x_{2} H_{02}^{y}(\mathbf{y})-x_{0} G_{2}^{y}(\mathbf{y}),-x_{2} H_{12}^{y}(\mathbf{y})-x_{1} G_{2}^{y}(\mathbf{y}), x_{2} G_{2}^{y}(\mathbf{y})\right]
$$

Return $\left(\left[x_{0}^{\prime}, x_{1}^{\prime}, x_{2}^{\prime}\right],\left[y_{0}, y_{1}, y_{2}\right]\right)$

\section{Algorithm to compute $\phi$ and $\psi$.}

Input $P=\left(\left[x_{0}, x_{1}, x_{2}\right],\left[y_{0}, y_{1}, y_{2}\right]\right) \quad$ (point on $\mathrm{S}$ )

$\phi(P)=\sigma^{y}\left(\sigma^{x}(P)\right)$

$\psi(P)=\sigma^{x}\left(\sigma^{y}(P)\right)$

Return $\phi(P)$ and $\psi(P)$

\section{Algorithm to compute $\hat{\lambda}^{+}$.}

Input $P=\left(\left[x_{0}, x_{1}, x_{2}\right],\left[y_{0}, y_{1}, y_{2}\right]\right) \quad$ (point on $\mathrm{S}$ )

Input $N \quad$ (number of terms to compute)

Input $m, n \quad$ (compute local height for the divisor $E_{m n}^{+}$)

Select $i$ with $\left|x_{i}\right|=\max \left\{\left|x_{0}\right|,\left|x_{1}\right|,\left|x_{2}\right|\right\}$

Select $j$ with $\left|y_{j}\right|=\max \left\{\left|y_{0}\right|,\left|y_{1}\right|,\left|y_{2}\right|\right\}$

LocalHeight $=\beta \log \left|\frac{x_{i}}{x_{m}}\right|-\log \left|\frac{y_{j}}{y_{n}}\right|$

Loop $e=0$ to $N-1$

Compute $Q=\left(\left[x_{0}^{\prime \prime}, x_{1}^{\prime \prime}, x_{2}^{\prime \prime}\right],\left[y_{0}^{\prime}, y_{1}^{\prime}, y_{2}^{\prime}\right]\right)=\phi(P)$

Select $k$ with $\left|x_{k}^{\prime \prime}\right|=\max \left\{\left|x_{0}^{\prime \prime}\right|,\left|x_{1}^{\prime \prime}\right|,\left|x_{2}^{\prime \prime}\right|\right\}$

Select $l$ with $\left|y_{l}^{\prime}\right|=\max \left\{\left|y_{0}^{\prime}\right|,\left|y_{1}^{\prime}\right|,\left|y_{2}^{\prime}\right|\right\}$

If $\left|y_{j}\right| \leq\left|y_{l}\right|$

$$
B=G_{l}^{x}\left(\frac{x_{0}}{x_{i}}, \frac{x_{1}}{x_{i}}, \frac{x_{2}}{x_{i}}\right) \cdot \frac{y_{j}}{y_{l}}
$$


Else $\left|y_{j}\right|>\left|y_{l}\right|$

End If

$$
B=-G_{j}^{x}\left(\frac{x_{0}}{x_{i}}, \frac{x_{1}}{x_{i}}, \frac{x_{2}}{x_{i}}\right) \frac{y_{l}}{y_{j}}-H_{j l}^{x}\left(\frac{x_{0}}{x_{i}}, \frac{x_{1}}{x_{i}}, \frac{x_{2}}{x_{i}}\right)
$$

If $\left|x_{i}\right| \leq\left|x_{k}\right|$

$$
A=G_{k}^{y}\left(\frac{y_{0}^{\prime}}{y_{l}^{\prime}}, \frac{y_{1}^{\prime}}{y_{l}^{\prime}}, \frac{y_{2}^{\prime}}{y_{l}^{\prime}}\right) \cdot \frac{x_{i}}{x_{k}}
$$

Else $\left|x_{i}\right|>\left|x_{k}\right|$

End If

$$
A=-G_{i}^{y}\left(\frac{y_{0}^{\prime}}{y_{l}^{\prime}}, \frac{y_{1}^{\prime}}{y_{l}^{\prime}}, \frac{y_{2}^{\prime}}{y_{l}^{\prime}}\right) \cdot \frac{x_{k}}{x_{i}}-H_{i k}^{y}\left(\frac{y_{0}^{\prime}}{y_{l}^{\prime}}, \frac{y_{1}^{\prime}}{y_{l}^{\prime}}, \frac{y_{2}^{\prime}}{y_{l}^{\prime}}\right)
$$

LocalHeight $=$ LocalHeight $+\beta^{-2 e-1} \log |A|+\beta^{-2 e} \log |B|$ $i=k: j=l: P=Q$

End Loop

Return LocalHeight

\section{Algorithm to compute $\hat{\lambda}^{-}$.}

Input $P=\left(\left[x_{0}, x_{1}, x_{2}\right],\left[y_{0}, y_{1}, y_{2}\right]\right) \quad$ (point on $\mathrm{S}$ )

Input $N \quad$ (number of terms to compute)

Input $m, n \quad$ (compute local height for the divisor $E_{m n}^{-}$)

Select $i$ with $\left|y_{i}\right|=\max \left\{\left|y_{0}\right|,\left|y_{1}\right|,\left|y_{2}\right|\right\}$

Select $j$ with $\left|x_{j}\right|=\max \left\{\left|x_{0}\right|,\left|x_{1}\right|,\left|x_{2}\right|\right\}$

LocalHeight $=\beta \log \left|\frac{y_{i}}{y_{m}}\right|-\log \left|\frac{x_{j}}{x_{n}}\right|$

Loop $e=0$ to $N-1$

Compute $Q=\left(\left[x_{0}^{\prime}, x_{1}^{\prime}, x_{2}^{\prime}\right],\left[y_{0}^{\prime \prime}, y_{1}^{\prime \prime}, y_{2}^{\prime \prime}\right]\right)=\phi^{-1}(P)$

Select $k$ with $\left|y_{k}^{\prime \prime}\right|=\max \left\{\left|y_{0}^{\prime \prime}\right|,\left|y_{1}^{\prime \prime}\right|,\left|y_{2}^{\prime \prime}\right|\right\}$

Select $l$ with $\left|x_{l}^{\prime}\right|=\max \left\{\left|x_{0}^{\prime}\right|,\left|x_{1}^{\prime}\right|,\left|x_{2}^{\prime}\right|\right\}$

If $\left|x_{j}\right| \leq\left|x_{l}\right|$

$$
B=G_{l}^{y}\left(\frac{y_{0}}{y_{i}}, \frac{y_{1}}{y_{i}}, \frac{y_{2}}{y_{i}}\right) \cdot \frac{x_{j}}{x_{l}}
$$

Else $\left|x_{j}\right|>\left|x_{l}\right|$

End If

$$
B=-G_{j}^{y}\left(\frac{y_{0}}{y_{i}}, \frac{y_{1}}{y_{i}}, \frac{y_{2}}{y_{i}}\right) \frac{x_{l}}{x_{j}}-H_{j l}^{y}\left(\frac{y_{0}}{y_{i}}, \frac{y_{1}}{y_{i}}, \frac{y_{2}}{y_{i}}\right)
$$

If $\left|y_{i}\right| \leq\left|y_{k}\right|$

$$
A=G_{k}^{x}\left(\frac{x_{0}^{\prime}}{x_{l}^{\prime}}, \frac{x_{1}^{\prime}}{x_{l}^{\prime}}, \frac{x_{2}^{\prime}}{x_{l}^{\prime}}\right) \cdot \frac{y_{i}}{y_{k}}
$$

Else $\left|y_{i}\right|>\left|y_{k}\right|$

$$
A=-G_{i}^{x}\left(\frac{x_{0}^{\prime}}{x_{l}^{\prime}}, \frac{x_{1}^{\prime}}{x_{l}^{\prime}}, \frac{x_{2}^{\prime}}{x_{l}^{\prime}}\right) \cdot \frac{y_{k}}{y_{i}}-H_{i k}^{x}\left(\frac{x_{0}^{\prime}}{x_{l}^{\prime}}, \frac{x_{1}^{\prime}}{x_{l}^{\prime}}, \frac{x_{2}^{\prime}}{x_{l}^{\prime}}\right)
$$

End If

LocalHeight $=$ LocalHeight $+\beta^{-2 e-1} \log |A|+\beta^{-2 e} \log |B|$ $i=k: j=l: P=Q$

End Loop

Return LocalHeight 


\section{REFERENCES}

1. G. Call, Geometry and heights on certain K3 surfaces, in preparation.

2. G. Call and J.H. Silverman, Canonical heights on varieties with morphisms, Compositio Math. 89 (1993), 163-205. MR 95d:11077

3. S. Lang, Fundamentals of Diophantine geometry, Springer-Verlag, New York, 1983. MR 85j: 11005

4. J.H. Silverman, The arithmetic of elliptic curves. I, Graduate Texts in Math., vol. 106, Springer-Verlag, Berlin and New York, 1986. MR 87g:11070

5. _ Computing heights on elliptic curves, Math. Comp. 51 (1988), 339-358. MR 89d: 11049

6. _ Computing heights on K3 surfaces: A new canonical height, Invent. Math. 105 (1991), 347-373.

7. J. Tate, Letter to J.-P. Serre, Oct. 1, 1979.

8. B.L. van der Waerden, Algebra, 7th ed., Ungar, New York, 1970. MR 41:8187

Department of Mathematics and Computer Science, Amherst College, Amherst, MasSACHUSETTS 01002

E-mail address: gscall@amherst.edu

Department of Mathematics, Box 1917, Brown University, Providence, Rhode Island 02912

E-mail address: jhs@gauss.math.brown.edu 